\title{
Contact analysis for dual peg-in-hole assembly of automobile alternator frame
}

\author{
Bo Zhou ${ }^{1}$, Lijun $\mathrm{Liu}^{2, *}$, and Guangyuan $\mathrm{Chen}^{3}$ \\ ${ }^{1}$ School of Mechanical Engineering, Zhejiang University, Hangzhou 310012, PR China \\ ${ }^{2}$ Ningbo Institude of Technology, Zhejiang University, Ningbo 315100, PR China \\ ${ }^{3}$ Ningbo Donghao Casting Co., Ltd., Ningbo 315113, PR China
}

Received: 17 April 2019 / Accepted: 30 January 2020

\begin{abstract}
The modeling and analysis of the assembly contact problems in the picking and placement of the automobile alternator frame are carried out in the multi-station processing. According to the three-dimensional assembly characteristics of the actual alternator frame and the fixture, the assembly model in the drilling and milling station is simplified. The alternator frame and fixture assembly problem as a whole is simplified to a dual peg-in-hole assembly problem. All possible one-point contact states and two-point contact states during the assembly process are proposed(L-1) (R-10). The contact force analysis is carried out on the typical one-contact state L-1 and the typical two-point contact state L-5, and the relationship between the corresponding assembly forces $F_{x}, F_{y}, F_{z}$ and the assembly moments $M_{x}, M_{y}, M_{z}$ is obtained. According to the relationship between these assembly forces and assembly moments, the corresponding jamming diagrams are obtained. Finally, according to the three-dimensional size of the actual alternator frame, the experimental verification was carried out by using a 6 -DOF force/moment sensor. The experimental results confirmed the validity of the analysis. Technical support is provided for the assembly of automobile alternator frames based on force sensing control for the follow-up work.
\end{abstract}

Keywords: Automobile alternator frame / model simplifying / contact state / contact force / dual peg-in-hole

\section{Introduction}

In the multi-station processing of automobile alternator frame, the picking and placing technology of the frame is a combination of mechanics, electronics and intelligent control technology. It is the key technology for assembling robots on the automated production line, and it is also the supportive technology for gripbing, handling, assembly, and palletizing of workpiece. However, the traditional assembly robot must be taught point by point to complete the "pick-place" action. The picking point, the placing point and the locus of the pick-place are all determined beforehand, so the automation production line must be highly structured. Moreover, traditional assembly robots require high precision of assembly environment and machine itself, have low assembly efficiency, lack of perception and adaptive control ability, and are difficult to complete complex assembly in changing environment. Especially, the workpieces on conveyor belt are moving and scattered, and the positions and postures of picking and

\footnotetext{
* e-mail: liulijunemail@163.com
}

placing points are generally not fixed, which greatly limits the application of traditional robots in modern flexible production lines. Therefore, using force sensing and human-like intelligent control technology, research on high-perception, high efficiency, high adaptability and high-precision assembly robot picking and placing technology has become one of the key technologies for processing and assembly of automobile alternator frame. In force sensing, the process of the robot gripbing the frame to the corresponding fixture can be regarded as the assembly of dual peg-in-hole. Therefore, it is necessary to analyze the characteristics of the process and simplify the model, and then to analyze the contact state and contact force. In the research of dual peg-in-hole assembly, domestic and foreign scholars have carried out certain researches. Based on the enumeration method, Korntham et al. analyzed jamming conditions in detail for dual peg-in-hole assembly [1]. However, this enumeration method is no longer applicable for multi peg-in-hole assembly with uncertain number. ARAI described the two-dimensional dual peg-in-hole assembly error model and analyzed the search strategy [2]. Sturges and Laowattana analyzed the conditions for inserting three-dimensional rectangular peg $[3,4]$. Fei 
et al. analyzed the three-dimensional dual peg-in-hole with the space screw theory and obtained the corresponding jamming diagrams [5]. However, there is no detailed analysis of the specific assembly strategy. Zhang et al. analyzed the assembly of flexible dual peg-in-hole and put forward the corresponding force control strategy, and made a series of analysis and research on rigid dual peg-in-hole $[6,7]$. However, as for the assembly strategy of the rigid dual peg-in-hole, they only use traditional impedance control to complete the assembly, but do not use jamming diagrams to guide the assembly, so that the adaptability is not high.

To the best of our knowledge, there is no research on the use of jamming diagrams to guide the assembly of the rigid dual peg-in-hole. On the basis of the former researches, this paper applies the related theory to the assembly of automobile alternator frame and fixture, abstracts and simplifies the assembly process model, and then makes corresponding analysis and research, finally, the corresponding assembly strategy is obtained.

\section{Simplification of assembly model}

\subsection{Analysis of assembly characteristics}

During the processing of the automobile alternator frame, after the frame blank is precision-cast-molded, it is processed through a series of rough processing such as removal handle, grinding, punching and trimming, and then the formed blanks are passed through the production line for turning, milling end faces, drilling and tapping of threaded holes and final cleaning operations. The entire production line only needs to put the rough-finished frame into the feeding conveying equipment, and then through the automatic control system, the robot grips the frame to successively enter the turning, milling, drilling and tapping, cleaning station for processing. After the processing is completed, the frame enters the detection module through the discharge conveyor belt for product qualification detection [8].

In the milling, drilling and tapping station, the assembly is carried out in the vertical direction. As shown in Figure 1, the experimental system is composed of the end of the system (1), the force acquisition system (2), the display (3), and the YASKAWA robot body system (4).

The detailed diagram of the end of the system (1) is shown in Figure 2. It consists of No. 1 connecting plate (1.1), No. 2 connecting plate (1.2), six-dimensional force sensor (1.3), No. 3 connecting plate (1.4), No. 4 connecting plate (1.5), grab device (1.6), alternator frame (1.7), fixture (1.8), etc.

After the grip part grips the alternator frame into the corresponding fixture, and the fixture fixes the frame, the machining center performs milling, drilling and tapping. In this assembly process, the position of the alternator frame is usually not fixed. If there is a positional deviation between the frame and the fixture, the frame will be stuck outside the fixture, and in severe cases, the gripping part will be damaged, so it is necessary to model the contact force for this process, which will be simplified and modeled below.

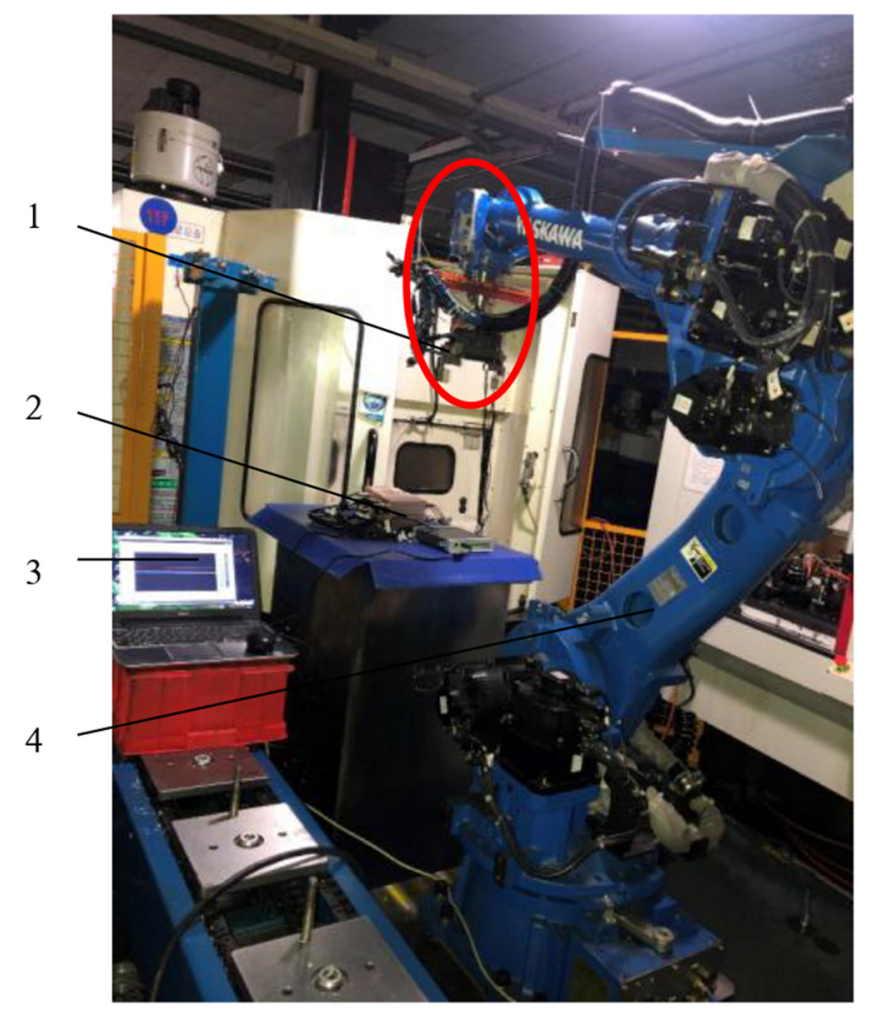

Fig. 1. Automobile alternator frame assembly system.

\subsection{Simplification of assembly model}

As shown in Figure 3, the alternator frame has two locating holes, which are respectively matched with the locating pins of the fixture during assembly. The physical diagram of the fixture is shown in Figure 4, in which the locating pin 1 matches with the locating hole 1 , the locating pin 2 matches with the locating hole 2 . The three support pins are of same height and form a horizontal plane for auxiliary fixing, which makes the frame relatively stable and prepares for fixing the frame. Therefore, in the milling, drilling and tapping station, the process of the robotic end effector gripping the frame into the corresponding fixture can be regarded as a dual peg-in-hole assembly. The simplified frame and fixture are shown in Figure 5. The radius of the left locating pin of the fixture is $r_{\mathrm{P} 1}$, and the radius of the right locating pin is $r_{\mathrm{P} 2}$; the radius of the left locating hole of the frame is $r_{\mathrm{H} 1}$, and the radius of the right locating hole is $r_{\mathrm{H} 2}$; the distance between the axes of the two locating pins is $D\left(D_{\mathrm{P}}\right)$, and the distance between the axes of the two locating holes is $D_{\mathrm{H}}$. It is considered that the locating pin and the locating hole have the same length. The coordinate system is set at the midpoint of the connection of the midpoints of the two locating holes of the frame on the end face. The $Z$ axis direction is downward perpendicular to the end face, and the $X$ axis direction is parallel to the connection of the midpoints of the two locating holes to the right. The $Y$ axis is determined according to the right-hand helix rule. In addition, in order to facilitate the analysis, we make the 


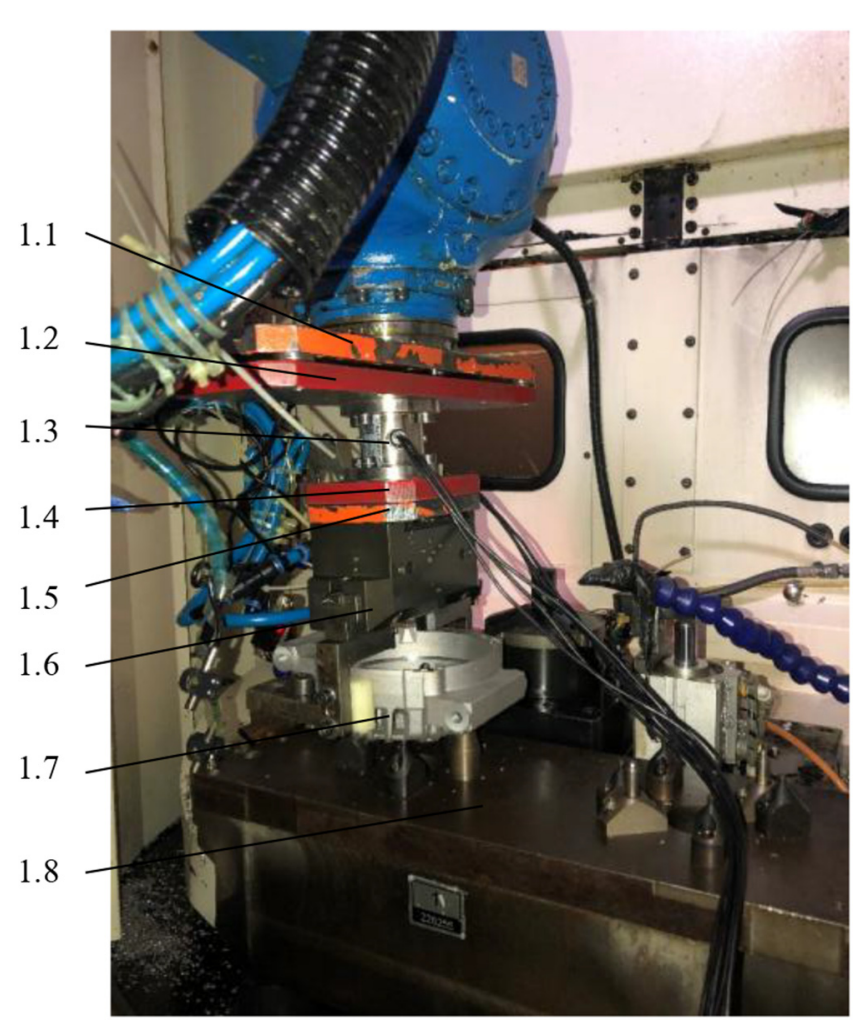

Fig. 2. End of the assembly system.

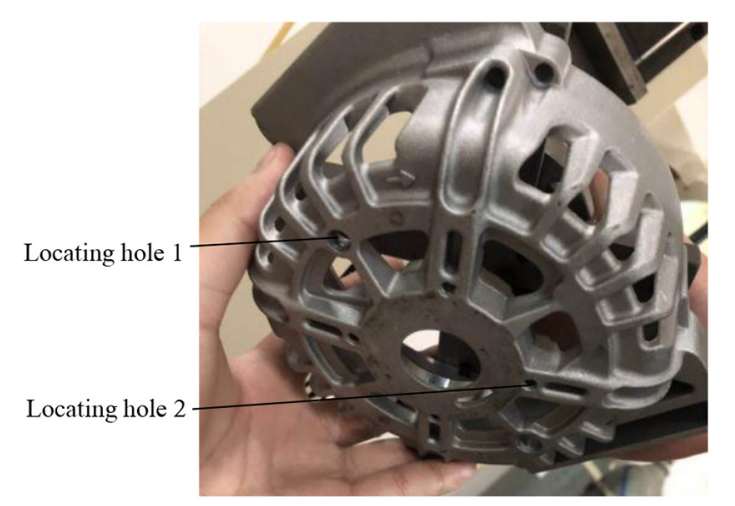

Fig. 3. Physical drawing of the frame.

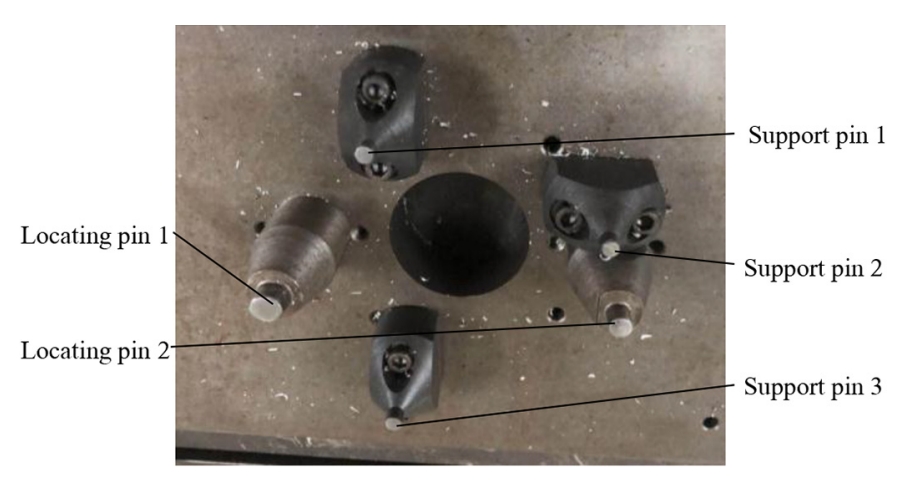

Fig. 4. Physical drawing of the fixture.

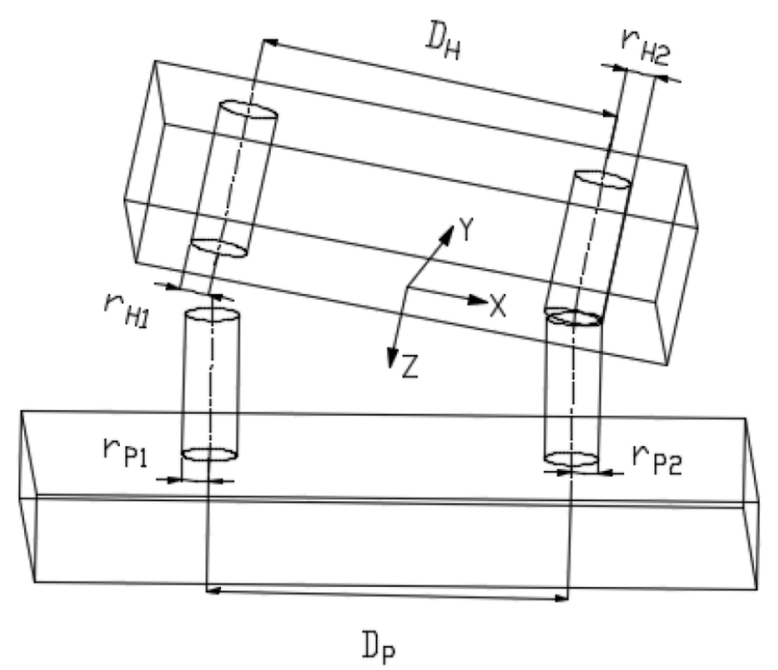

Fig. 5. Simplified model for assembly of fixture and frame.

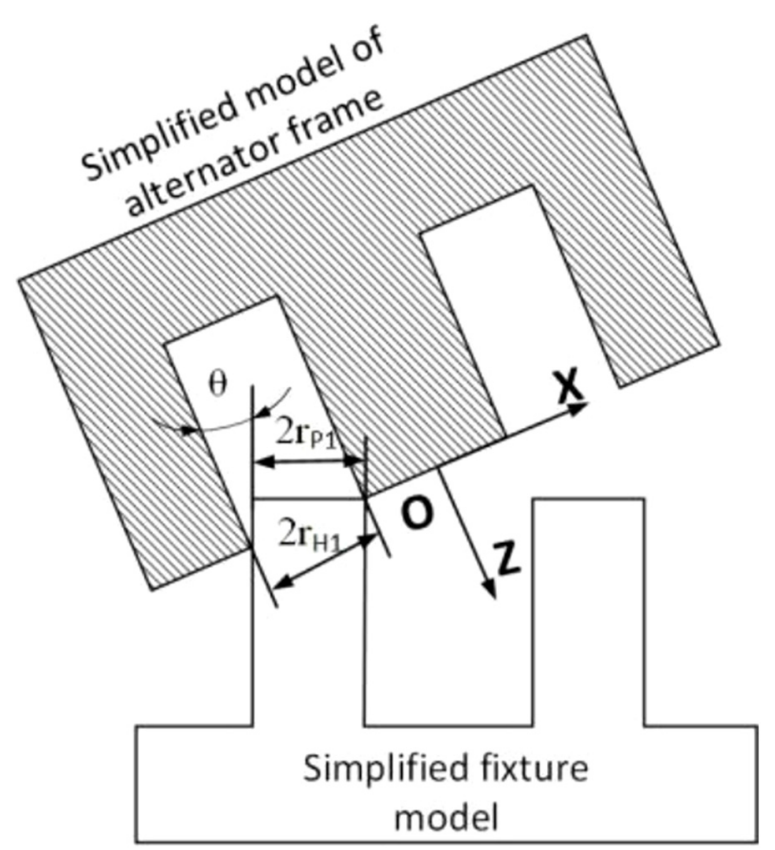

Fig. 6. Critical mode of contact state L-1.

following assumptions:

- In the process of assembling, certain geometric conditions must be met when the locating holes and pins are assembled: as shown in Figure 6, the acute angle $\theta$ between the axis of locating hole and the axis of the locating pin must meet $0 \leq \theta \leq\left\{\arccos \frac{r_{\mathrm{P} 1}}{r_{\mathrm{H} 1}}, \arccos \frac{r_{\mathrm{P} 2}}{r_{\mathrm{H} 2}}\right\}_{\text {min }}$, and the radius of the locating hole and the radius of the locating pin are usually close, so $\theta$ is small and negligible.

- Assume that the assembly is done in the vertical direction. 
- The locating pins have the same length and the axes are parallel to each other and perpendicular to the horizontal plane.

- Assembly process is a quasi-static process.

\section{Contact state analysis}

Due to various geometric and control uncertainties in the multi-station processing of the frames, various contact states exist during the assembly process. For the drilling and milling station, the model simplification result is a dual peg-in-hole assembly. Only when the locating holes of the frame and the corresponding locating pins of the fixture meet certain conditions, the three-point or four-point contact state exists, so these contacts are only temporary and cannot be sustained. In this paper, only the conditions for the one-point contact state and the two-point contact state are derived and analyzed.

During the assembly process, when the frame is tilted to the left, there will be ten point contact states, as shown in Table 1. It lists all possible point contact conditions for the frame during assembly. By mapping the contact force vector $f_{i}$ to the $X O Y$ plane, the corresponding vector $f_{i}^{*}$ can be obtained, and $\alpha_{i}$ represents the angle formed by the contact force vector projection $f_{i}^{*}$ and the $X$ axis forward direction. According to the previous assumption, $\theta$ is negligible, so $\alpha_{i}$ is equal to the angle formed by $f_{i}$ and $X$ axis forward direction. $r_{i}$ represents the vector formed by the contact point and origin $O, h_{i}$ represents the distance from the contact point of the frame and fixture to the origin $O$ in the $Z$ axis direction during assembly, and the friction coefficient between the frame and fixture is $\mu$. $i=1$ represents that the left locating hole of the frame contacts with the left locating pin of the fixture on the left, $i=2$ represents that the left locating hole of the frame contacts with the left locating pin of the fixture on the right, $i=3$ represents that the right locating hole of the frame contacts with the right locating pin of the fixture on the left, and $i=4$ represents that the right locating hole of the frame contacts with the right locating pin of the fixture on the right. $\mathrm{L}$ means the frame is tilted to the left and $\mathrm{R}$ means the frame is tilted to the right.

\section{Contact force analysis}

By understanding the characteristics of the forces and moments during the assembly process of the frame, the corresponding assembly strategy can be well developed to make the assembly successful, and in this process, the assembly forces and moments acting on the frame $F_{x}, F_{y}$, $F_{z}, M_{x}, M_{y}$ and $M_{z}$ must meet certain conditions, so it is necessary to derive the conditions of force and moment to be met for each contact state.

As mentioned above, we only analyze one-point contact states and two-point contact states, while the multi-point contact state is neglected because it is temporary. Because the assembly process is assumed to be quasi-static, the relationship between force and moment can be obtained by using static equilibrium equation. The following is a detailed analysis of one-point contact state (L-1) as shown in Figure 7a and two-point contact state (L-5) as shown in Figure $7 \mathrm{~b}$, and other contact states can be analogous. Table 2 lists the relationship between the forces and moments of all possible contact states of the frame, where $c \alpha_{i}$ represents $\cos \alpha_{i}$ and $\mathrm{s} \alpha_{i}$ represents $\sin \alpha_{i}$.

\subsection{One point contact state L-1}

A two-dimensional simplified diagram of the one-point contact state L-1 is shown in Figure 7a. The range of the contact force acting on the left locating hole of the frame is as shown in the range of the solid arrow in Figure 8, that is, the left half of the left locating hole. Let one of them be $f_{1}$, and the angle between it and the positive $X$ axis is $\alpha_{1}$, the friction is $\mu f_{1}$, the direction is vertical to the paper facing outward, and the assembly forces and moments are $F_{x}, F_{y}, F_{z}, M_{x}, M_{y}$, and $M_{z}$ respectively, that is, the forces and moments measured by the 6 -DOF force/moment sensor. According to the static equilibrium equation, we can get:

$$
\left\{\begin{array}{l}
F_{x}+f_{1} \cos \alpha_{1}=0 \\
F_{y}+f_{1} \sin \alpha_{1}=0 \\
F_{z}-\mu f_{1}=0 \\
M_{x}+\mu f_{1} y_{1}=0 \\
M_{y}-\mu f_{1} x_{1}=0 \\
M_{z}+f_{1} y_{1} \cos \alpha_{1}-f_{1} x_{1} \sin \alpha_{1}=0
\end{array}\right.
$$

From (1), the following relationship between force and moment can be obtained:

$$
\begin{aligned}
& \left\{\begin{array}{l}
\frac{F_{x}}{F_{z}}=-\frac{\mathrm{c} \alpha_{1}}{\mu} \\
\frac{M_{y}}{F_{z}}=x_{1}
\end{array}\right. \\
& \left\{\begin{array}{l}
\frac{F_{y}}{F_{z}}=-\frac{\mathrm{s} \alpha_{1}}{\mu} \\
\frac{M_{x}}{F_{z}}=-y_{1}
\end{array}\right.
\end{aligned}
$$

\subsection{Two-point contact state L-5}

The two-dimensional simplified diagram of the two-point contact state L-5 is shown in Figure 7b. The contact force acting on the left locating hole of the frame is $f_{1}$ and $f_{2}$, of which the description of $f_{1}$ is the same as above, and the range of the action points of $f_{2}$ is shown by the dotted arrow of Figure 9, that is, the right half of the left locating hole. Let one of them be $f_{2}$, and the angle between it and the positive direction of the $X$ axis is $\alpha_{2}$. The friction is $\mu f_{1}$ and $\mu f_{2}$, the direction is vertical to the paper facing outward, and the assembly forces and moments are $F_{x}, F_{y}, F_{z}, M_{x}, M_{y}$ and $M_{z}$ respectively, as shown in Figure 9 . According to the 
Table 1. Three-dimensional assembly contact state of the frame.

\begin{tabular}{|c|c|c|c|}
\hline$\overline{\text { Model }}$ & $\begin{array}{l}\text { Three-dimensional contact } \\
\text { state }\end{array}$ & $\alpha_{i}$ & $r_{i}$ \\
\hline \multirow[t]{12}{*}{ Frame tilted to the left } & One-point contact (L-1) & $\alpha_{1} \in\left[90^{\circ}, 270^{\circ}\right]$ & $r_{1}:\left(x_{1}, y_{1}, 0\right)$ \\
\hline & $(\mathrm{L}-2)$ & $\alpha_{2} \in\left[0^{\circ}, 90^{\circ}\right] \cup\left[270^{\circ}, 360^{\circ}\right]$ & $r_{2}:\left(x_{2}, y_{2},-h_{2}\right)$ \\
\hline & $(\mathrm{L}-3)$ & $\alpha_{3} \in\left[90^{\circ}, 270^{\circ}\right]$ & $r_{3}:\left(x_{3}, y_{3}, 0\right)$ \\
\hline & $(\mathrm{L}-4)$ & $\alpha_{4} \in\left[0^{\circ}, 90^{\circ}\right] \cup\left[270^{\circ}, 360^{\circ}\right]$ & $r_{4}:\left(x_{4}, y_{4},-h_{4}\right)$ \\
\hline & Two-point contact (L-5) & $\alpha_{1} \in\left[90^{\circ}, 270^{\circ}\right], \alpha_{2} \in\left[0^{\circ}, 90^{\circ}\right] \cup\left[270^{\circ}, 360^{\circ}\right]$ & $r_{1}:\left(x_{1}, y_{1}, 0\right)$ \\
\hline & & & $r_{2}:\left(x_{2}, y_{2},-h_{2}\right)$ \\
\hline & $(\mathrm{L}-6)$ & $\alpha_{3} \in\left[90^{\circ}, 270^{\circ}\right], \alpha_{4} \in\left[0^{\circ}, 90^{\circ}\right] \cup\left[270^{\circ}, 360^{\circ}\right]$ & $\begin{array}{l}r_{3}:\left(x_{3}, y_{3}, 0\right) \\
r_{4}:\left(x_{1}, y_{4},-h_{4}\right)\end{array}$ \\
\hline & $(\mathrm{L}-7)$ & $\alpha_{1} \in\left[90^{\circ}, 270^{\circ}\right], \alpha_{3} \in\left[90^{\circ}, 270^{\circ}\right]$ & $r_{1}:\left(x_{1}, y_{1}, 0\right)$ \\
\hline & $\left(\mathrm{L}_{-}-8\right)$ & $\alpha_{1} \in\left[90^{\circ}, 270^{\circ}\right], \alpha_{1} \in\left[0^{\circ}, 90^{\circ}\right] \cup\left[270^{\circ}, 360^{\circ}\right]$ & $\begin{array}{l}r_{3}:\left(x_{3}, y_{3}, 0\right) \\
r_{1}:\left(x_{1}, y_{1}, 0\right)\end{array}$ \\
\hline & $(L-0)$ & $\alpha_{1} \in[90,260], \alpha_{4} \in[0,90] \cup[260,360]$ & $r_{4}:\left(x_{4}, y_{4},-h_{4}\right)$ \\
\hline & $(\mathrm{L}-9)$ & $\alpha_{2} \in\left[0^{\circ}, 90^{\circ}\right] \cup\left[270^{\circ}, 360^{\circ}\right], \alpha_{3} \in\left[90^{\circ}, 270^{\circ}\right]$ & $r_{2}:\left(x_{2}, y_{2},-h_{2}\right)$ \\
\hline & $(\mathrm{L}-10)$ & $\alpha_{2} \in\left[0^{\circ}, 90^{\circ}\right] \cup\left[270^{\circ}, 360^{\circ}\right], \alpha_{4} \in\left[0^{\circ}, 90^{\circ}\right] \cup\left[270^{\circ}, 360^{\circ}\right]$ & $\begin{array}{l}r_{3}:\left(x_{3}, y_{3}, 0\right) \\
r_{2}:\left(x_{2}, y_{2},-h_{2}\right) \\
r_{4}:\left(x_{4}, y_{4},-h_{4}\right)\end{array}$ \\
\hline \multirow[t]{12}{*}{ Frame tilted to the right } & One-point contact (R-1) & $\alpha_{1} \in\left[90^{\circ}, 270^{\circ}\right]$ & $\begin{array}{l}r_{4}:\left(x_{4}, y_{4},-h_{4}\right) \\
r_{1}:\left(x_{1}, y_{1},-h_{1}\right)\end{array}$ \\
\hline & $(\mathrm{R}-2)$ & $\alpha_{2} \in\left[0^{\circ}, 90^{\circ}\right] \cup\left[270^{\circ}, 360^{\circ}\right]$ & $r_{2}:\left(x_{2}, y_{2}, 0\right)$ \\
\hline & $(\mathrm{R}-3)$ & $\alpha_{3} \in\left[90^{\circ}, 270^{\circ}\right]$ & $r_{3}:\left(x_{3}, y_{3},-h_{3}\right)$ \\
\hline & $(\mathrm{R}-4)$ & $\alpha_{4} \in\left[0^{\circ}, 90^{\circ}\right] \cup\left[270^{\circ}, 360^{\circ}\right]$ & $r_{4}:\left(x_{4}, y_{4}, 0\right)$ \\
\hline & Two-point contact (R-5) & $\alpha_{1} \in\left[90^{\circ}, 270^{\circ}\right], \alpha_{2} \in\left[0^{\circ}, 90^{\circ}\right] \cup\left[270^{\circ}, 360^{\circ}\right]$ & $r_{1}:\left(x_{1}, y_{1},-h_{1}\right)$ \\
\hline & $(\mathrm{R}-6)$ & $\alpha_{3} \in\left[90^{\circ}, 270^{\circ}\right], \alpha_{4} \in\left[0^{\circ}, 90^{\circ}\right] \cup\left[270^{\circ}, 360^{\circ}\right]$ & $\begin{array}{l}r_{2}:\left(x_{2}, y_{2}, 0\right) \\
r_{3}:\left(x_{3}, y_{3},-h_{3}\right)\end{array}$ \\
\hline & $(\mathrm{R}-7)$ & $\alpha_{1} \in\left[90^{\circ}, 270^{\circ}\right], \alpha_{3} \in\left[90^{\circ}, 270^{\circ}\right]$ & $\begin{aligned} r_{4} & :\left(x_{4}, y_{4}, 0\right) \\
r_{1} & :\left(x_{1}, y_{1},-h_{1}\right) \\
r_{3} & :\left(x_{3}, y_{3},-h_{3}\right)\end{aligned}$ \\
\hline & $(\mathrm{R}-8)$ & $\alpha_{1} \in\left[90^{\circ}, 270^{\circ}\right], \alpha_{4} \in\left[0^{\circ}, 90^{\circ}\right] \cup\left[270^{\circ}, 360^{\circ}\right]$ & $r_{1}:\left(x_{1}, y_{1},-h_{1}\right)$ \\
\hline & $(\mathrm{R}-9)$ & $\alpha_{2} \in\left[0^{\circ}, 90^{\circ}\right] \cup\left[270^{\circ}, 360^{\circ}\right], \alpha_{3} \in\left[90^{\circ}, 270^{\circ}\right]$ & $\begin{array}{l}r_{4}:\left(x_{4}, y_{4}, 0\right) \\
r_{2}:\left(x_{2}, y_{2}, 0\right)\end{array}$ \\
\hline & & & $r_{3}:\left(x_{3}, y_{3},-h_{3}\right)$ \\
\hline & $(\mathrm{R}-10)$ & $\alpha_{2} \in\left[0^{\circ}, 90^{\circ}\right] \cup\left[270^{\circ}, 360^{\circ}\right], \alpha_{4} \in\left[0^{\circ}, 90^{\circ}\right] \cup\left[270^{\circ}, 360^{\circ}\right]$ & $r_{2}:\left(x_{2}, y_{2}, 0\right)$ \\
\hline & & & $r_{4}:\left(x_{4}, y_{4}, 0\right)$ \\
\hline
\end{tabular}

static equilibrium equation, we can get:

$$
\left\{\begin{array}{l}
F_{x}+f_{1} \cos \alpha_{1}+f_{2} \cos \alpha_{2}=0 \\
F_{y}+f_{1} \sin \alpha_{1}+f_{2} \sin \alpha_{2}=0 \\
F_{z}-\mu f_{1}-\mu f_{2}=0 \\
M_{x}+\mu f_{1} y_{1}-f_{2} h_{2} \sin \alpha_{2}+\mu f_{2} y_{2}=0 \\
M_{y}-\mu f_{1} x_{1}+f_{2} h_{2} \cos \alpha_{2}-\mu f_{2} x_{2}=0 \\
M_{z}+f_{1} y_{1} \cos \alpha_{1}-f_{1} x_{1} \sin \alpha_{1}+f_{2} y_{2} \cos \alpha_{2}-f_{2} x_{2} \sin \alpha_{2}=0
\end{array}\right.
$$

From (4), we can get:

$$
\begin{aligned}
\frac{M_{y}}{D F_{z}}= & -\frac{\mu}{\mathrm{c} \alpha_{1}-\mathrm{c} \alpha_{2}}\left(\frac{x_{1}-x_{2}}{D}+\frac{h_{2} \mathrm{c} \alpha_{2}}{\mu D}\right) \frac{F_{x}}{F_{z}} \\
& +\frac{\mu x_{1} \mathrm{c} \alpha_{2}-\mu x_{2} \mathrm{c} \alpha_{1}+h_{2} \mathrm{c} \alpha_{1} \mathrm{c} \alpha_{2}}{\mu D\left(\mathrm{c} \alpha_{2}-\mathrm{c} \alpha_{1}\right)} \\
\frac{M_{x}}{D F_{z}}= & -\frac{\mu}{\mathrm{s} \alpha_{2}-\mathrm{s} \alpha_{1}}\left(\frac{y_{1}-y_{2}}{D}+\frac{h_{2} \mathrm{~s} \alpha_{2}}{\mu D}\right) \frac{F_{y}}{F_{z}} \\
& +\frac{\mu y_{1} \mathrm{~s} \alpha_{2}-\mu y_{2} \mathrm{~s} \alpha_{1}+h_{2} \mathrm{~s} \alpha_{1} \mathrm{~s} \alpha_{2}}{\mu D\left(\mathrm{~s} \alpha_{1}-\mathrm{s} \alpha_{2}\right)}
\end{aligned}
$$




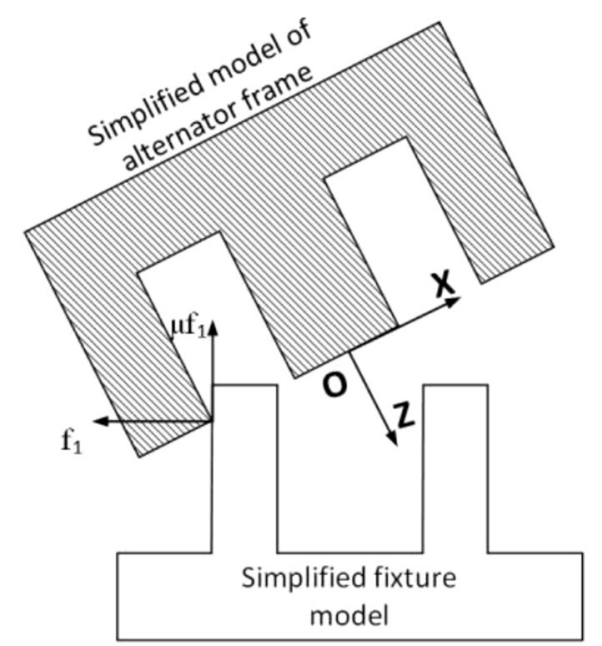

(a) State L-1 $\left(\alpha_{1}=180^{\circ}\right)$

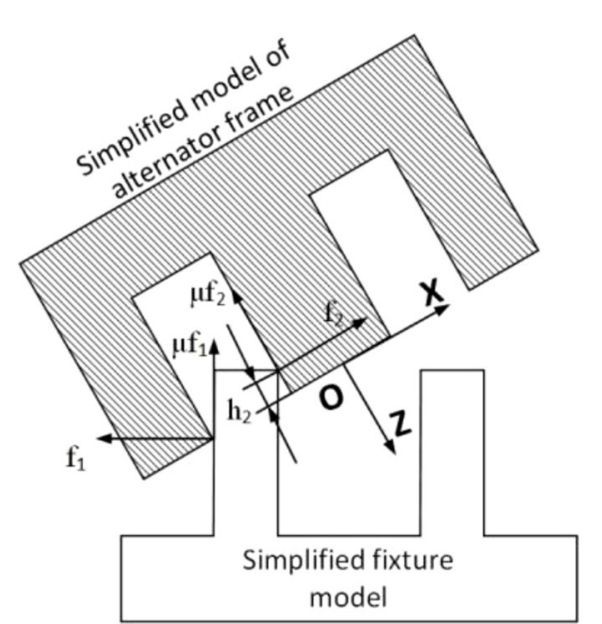

(b) State $\mathrm{L}-5 \quad\left(\alpha_{1}=180^{\circ}, \alpha_{2}=0^{\circ}\right)$

Fig. 7. 2-D simplified assembly diagram of the frame.

Table 2. Three-dimensional contact assembly force and moment relationship of the frame.

$$
\begin{aligned}
& \text { Contact Assembly force and moment relation } \\
& \text { state } \\
& \text { L-1 } \\
& \left\{\begin{array}{l}
\frac{F_{x}}{F_{z}}=-\frac{\mathrm{c} \alpha_{1}}{\mu} \\
\frac{M_{y}}{F_{z}}=x_{1}
\end{array},\left\{\begin{array}{l}
\frac{F_{y}}{F_{z}}=-\frac{\mathrm{s} \alpha_{1}}{\mu} \\
\frac{M_{x}}{F_{z}}=-y_{1}
\end{array}\right.\right. \\
& \left\{\begin{array}{l}
\frac{F_{x}}{F_{z}}=-\frac{\mathrm{c} \alpha_{2}}{\mu} \\
\frac{M_{y}}{F_{z}}=-\frac{h_{2} \mathrm{c} \alpha_{2}}{\mu}+x_{2}
\end{array},\left\{\begin{array}{l}
\frac{F_{y}}{F_{z}}=-\frac{\mathrm{s} \alpha_{2}}{\mu} \\
\frac{M_{x}}{F_{z}}=\frac{h_{2} \mathrm{~s} \alpha_{2}}{\mu}-y_{2}
\end{array}\right.\right. \\
& \text { L-3 } \\
& \text { L-4 } \\
& \left\{\begin{array}{l}
\frac{F_{x}}{F_{z}}=-\frac{\mathrm{c} \alpha_{4}}{\mu} \\
\frac{M_{y}}{F_{z}}=-\frac{h_{4} \mathrm{c} \alpha_{4}}{\mu}+x_{4}
\end{array},\left\{\begin{array}{l}
\frac{F_{y}}{F_{z}}=-\frac{\mathrm{s} \alpha_{4}}{\mu} \\
\frac{M_{x}}{F_{z}}=\frac{h_{4} \mathrm{~s} \alpha_{4}}{\mu}-y_{4}
\end{array}\right.\right. \\
& \frac{M_{y}}{D F_{z}}=-\frac{\mu}{c \alpha_{1}-c \alpha_{2}}\left(\frac{x_{1}-x_{2}}{D}+\frac{h_{2} c \alpha_{2}}{\mu D}\right) \frac{F_{x}}{F_{z}} \\
& +\frac{\mu x_{1} \mathrm{c} \alpha_{2}-\mu x_{2} \mathrm{c} \alpha_{1}+h_{2} \mathrm{c} \alpha_{1} \mathrm{c} \alpha_{2}}{\mu D\left(\mathrm{c} \alpha_{2}-\mathrm{c} \alpha_{1}\right)} \\
& \frac{M_{x}}{D F_{z}}=-\frac{\mu}{\mathrm{s} \alpha_{2}-\mathrm{s} \alpha_{1}}\left(\frac{y_{1}-y_{2}}{D}+\frac{h_{2} \mathrm{~s} \alpha_{2}}{\mu D}\right) \frac{F_{y}}{F_{z}} \\
& +\frac{\mu y_{1} \mathrm{~s} \alpha_{2}-\mu y_{2} \mathrm{~s} \alpha_{1}+h_{2} \mathrm{~s} \alpha_{1} \mathrm{~s} \alpha_{2}}{\mu D\left(\mathrm{~s} \alpha_{1}-\mathrm{s} \alpha_{2}\right)}
\end{aligned}
$$

Table 2. (continued).

$$
\begin{aligned}
& \text { Contact Assembly force and moment relation } \\
& \text { state } \\
& \begin{aligned}
\frac{M_{y}}{D F_{z}}= & -\frac{\mu}{\mathrm{c} \alpha_{3}-\mathrm{c} \alpha_{4}}\left(\frac{x_{3}-x_{4}}{D}+\frac{h_{4} \mathrm{c} \alpha_{4}}{\mu D}\right) \frac{F_{x}}{F_{z}} \\
& +\frac{\mu x_{4} \mathrm{c} \alpha_{3}-\mu x_{3} \mathrm{c} \alpha_{4}-h_{4} \mathrm{c} \alpha_{3} \mathrm{c} \alpha_{4}}{\mu D\left(\mathrm{c} \alpha_{3}-\mathrm{c} \alpha_{4}\right)}
\end{aligned} \\
& \frac{M_{x}}{D F_{z}}=-\frac{\mu}{\mathrm{s} \alpha_{3}-\mathrm{s} \alpha_{4}}\left(\frac{y_{4}-y_{3}}{D}-\frac{h_{4} \mathrm{~s} \alpha_{4}}{\mu D}\right) \frac{F_{y}}{F_{z}} \\
& +\frac{\mu y_{3} \mathrm{~s} \alpha_{4}-\mu y_{4} \mathrm{~s} \alpha_{3}+h_{4} \mathrm{~s} \alpha_{3} \mathrm{~s} \alpha_{4}}{\mu D\left(\mathrm{~s} \alpha_{3}-\mathrm{s} \alpha_{4}\right)} \\
& \frac{M_{y}}{D F_{z}}=-\frac{\mu}{c \alpha_{1}-c \alpha_{3}}\left(\frac{x_{1}-x_{3}}{D}\right) \frac{F_{x}}{F_{z}}+\frac{\mu x_{3} \mathrm{c} \alpha_{1}-\mu x_{1} \mathrm{c} \alpha_{3}}{\mu D\left(\mathrm{c} \alpha_{1}-\mathrm{c} \alpha_{3}\right)} \\
& \frac{M_{x}}{D F_{z}}=-\frac{\mu}{\mathrm{s} \alpha_{1}-\mathrm{s} \alpha_{3}}\left(\frac{y_{3}-y_{1}}{D}\right) \frac{F_{y}}{F_{z}}+\frac{\mu y_{1} \mathrm{~s} \alpha_{3}-\mu y_{3} \mathrm{~s} \alpha_{1}}{\mu D\left(\mathrm{~s} \alpha_{1}-\mathrm{s} \alpha_{3}\right)} \\
& \begin{aligned}
\frac{M_{y}}{D F_{z}}= & -\frac{\mu}{\mathrm{c} \alpha_{1}-\mathrm{c} \alpha_{4}}\left(\frac{x_{1}-x_{4}}{D}+\frac{h_{4} \mathrm{c} \alpha_{4}}{\mu D}\right) \frac{F_{x}}{F_{z}} \\
& +\frac{\mu x_{4} \mathrm{c} \alpha_{1}-\mu x_{1} \mathrm{c} \alpha_{4}-h_{4} \mathrm{c} \alpha_{1} \mathrm{c} \alpha_{4}}{\mu D\left(\mathrm{c} \alpha_{1}-\mathrm{c} \alpha_{4}\right)}
\end{aligned} \\
& \frac{M_{x}}{D F_{z}}=-\frac{\mu}{\mathrm{s} \alpha_{1}-\mathrm{s} \alpha_{4}}\left(\frac{y_{4}-y_{1}}{D}-\frac{h_{4} \mathrm{~s} \alpha_{4}}{\mu D}\right) \frac{F_{y}}{F_{z}} \\
& +\frac{\mu y_{1} \mathrm{~s} \alpha_{4}-\mu y_{4} \mathrm{~s} \alpha_{1}+h_{4} \mathrm{~s} \alpha_{1} \mathrm{~s} \alpha_{4}}{\mu D\left(\mathrm{~s} \alpha_{1}-\mathrm{s} \alpha_{4}\right)} \\
& \frac{M_{y}}{D F_{z}}=-\frac{\mu}{\mathrm{c} \alpha_{2}-\mathrm{c} \alpha_{3}}\left(\frac{x_{2}-x_{3}}{D}-\frac{h_{2} c \alpha_{2}}{\mu D}\right) \frac{F_{x}}{F_{z}} \\
& +\frac{\mu x_{3} c \alpha_{2}-\mu x_{2} c \alpha_{3}+h_{2} c \alpha_{2} c \alpha_{3}}{\mu D\left(c \alpha_{2}-c \alpha_{3}\right)}
\end{aligned}
$$


Table 2. (continued).

Contact Assembly force and moment relation

state

L-9

$$
\begin{gathered}
\frac{M_{x}}{D F_{z}}=-\frac{\mu}{\mathrm{s} \alpha_{2}-\mathrm{s} \alpha_{3}}\left(\frac{y_{3}-y_{2}}{D}+\frac{h_{2} \mathrm{~s} \alpha_{2}}{\mu D}\right) \frac{F_{y}}{F_{z}} \\
+\frac{\mu y_{2} \mathrm{~s} \alpha_{3}-\mu y_{3} \mathrm{~s} \alpha_{2}-h_{2} \mathrm{~s} \alpha_{2} \mathrm{~s} \alpha_{3}}{\mu D\left(\mathrm{~s} \alpha_{2}-\mathrm{s} \alpha_{3}\right)} \\
\frac{M_{y}}{D F_{z}}=-\frac{\mu}{\mathrm{c} \alpha_{2}-\mathrm{c} \alpha_{4}}\left(\frac{x_{2}-x_{4}}{D}+\frac{h_{4} \mathrm{c} \alpha_{4}-h_{2} \mathrm{c} \alpha_{2}}{\mu D}\right) \frac{F_{x}}{F_{z}} \\
+\frac{\mu x_{4} \mathrm{c} \alpha_{2}-\mu x_{2} \mathrm{c} \alpha_{4}+\left(h_{2}-h_{4}\right) \mathrm{c} \alpha_{2} \mathrm{c} \alpha_{4}}{\mu D\left(c \alpha_{2}-\mathrm{c} \alpha_{4}\right)}
\end{gathered}
$$

L-10

$$
\begin{aligned}
\frac{M_{x}}{D F_{z}}= & -\frac{\mu}{\mathrm{s} \alpha_{2}-\mathrm{s} \alpha_{4}}\left(\frac{y_{4}-y_{2}}{D}+\frac{h_{2} \mathrm{~s} \alpha_{2}-h_{4} \mathrm{~s} \alpha_{4}}{\mu D}\right) \frac{F_{y}}{F_{z}} \\
& +\frac{\mu y_{2} \mathrm{~s} \alpha_{4}-\mu y_{4} \mathrm{~s} \alpha_{2}+\left(h_{4}-h_{2}\right) \mathrm{s} \alpha_{2} \mathrm{~s} \alpha_{4}}{\mu D\left(\mathrm{~s} \alpha_{2}-\mathrm{s} \alpha_{4}\right)}
\end{aligned}
$$

R-1

$$
\left\{\begin{array}{l}
\frac{F_{x}}{F_{z}}=-\frac{\mathrm{c} \alpha_{1}}{\mu} \\
\frac{M_{y}}{F_{z}}=-\frac{h_{1} \mathrm{c} \alpha_{1}}{\mu}+x_{1}
\end{array},\left\{\begin{array}{l}
\frac{F_{y}}{F_{z}}=-\frac{\mathrm{s} \alpha_{1}}{\mu} \\
\frac{M_{x}}{F_{z}}=\frac{h_{1} \mathrm{~s} \alpha_{1}}{\mu}-y_{1}
\end{array}\right.\right.
$$

R-2

$$
\left\{\begin{array}{l}
\frac{F_{x}}{F_{z}}=-\frac{\mathrm{c} \alpha_{2}}{\mu} \\
\frac{M_{y}}{F_{z}}=x_{2}
\end{array},\left\{\begin{array}{l}
\frac{F_{y}}{F_{z}}=-\frac{\mathrm{s} \alpha_{2}}{\mu} \\
\frac{M_{x}}{F_{z}}=-y_{2}
\end{array}\right.\right.
$$

R-3

$$
\left\{\begin{array}{l}
\frac{F_{x}}{F_{z}}=-\frac{\mathrm{c} \alpha_{3}}{\mu} \\
\frac{M_{y}}{F_{z}}=-\frac{h_{3} \mathrm{c} \alpha_{3}}{\mu}+x_{3}
\end{array},\left\{\begin{array}{l}
\frac{F_{y}}{F_{z}}=-\frac{\mathrm{s} \alpha_{3}}{\mu} \\
\frac{M_{x}}{F_{z}}=\frac{h_{3} \mathrm{~s} \alpha_{3}}{\mu}-y_{3}
\end{array}\right.\right.
$$

R-4

$$
\begin{aligned}
& \left\{\begin{array}{l}
\frac{F_{x}}{F_{z}}=-\frac{\mathrm{c} \alpha_{4}}{\mu} \\
\frac{M_{y}}{F_{z}}=x_{4}
\end{array},\left\{\begin{array}{l}
\frac{F_{y}}{F_{z}}=-\frac{\mathrm{s} \alpha_{4}}{\mu} \\
\frac{M_{x}}{F_{z}}=-y_{4}
\end{array}\right.\right. \\
& \frac{M_{y}}{D F_{z}}=-\frac{\mu}{c \alpha_{1}-c \alpha_{2}}\left(\frac{x_{1}-x_{2}}{D}-\frac{h_{1} c \alpha_{1}}{\mu D}\right) \frac{F_{x}}{F_{z}} \\
& +\frac{\mu x_{2} c \alpha_{1}-\mu x_{1} c \alpha_{2}+h_{1} c \alpha_{1} c \alpha_{2}}{\mu D\left(c \alpha_{1}-c \alpha_{2}\right)} \\
& \frac{M_{x}}{D F_{z}}=-\frac{\mu}{\mathrm{s} \alpha_{1}-\mathrm{s} \alpha_{2}}\left(\frac{y_{2}-y_{1}}{D}+\frac{h_{1} \mathrm{~s} \alpha_{1}}{\mu D}\right) \frac{F_{y}}{F_{z}} \\
& +\frac{\mu y_{1} \mathrm{~s} \alpha_{2}-\mu y_{2} \mathrm{~s} \alpha_{1}-h_{1} \mathrm{~s} \alpha_{1} \mathrm{~s} \alpha_{2}}{\mu D\left(\mathrm{~s} \alpha_{1}-\mathrm{s} \alpha_{2}\right)}
\end{aligned}
$$

R-5
Table 2. (continued).

Contact Assembly force and moment relation
state

$$
\begin{aligned}
\frac{M_{y}}{D F_{z}}= & -\frac{\mu}{\mathrm{c} \alpha_{3}-\mathrm{c} \alpha_{4}}\left(\frac{x_{3}-x_{4}}{D}-\frac{h_{3} \mathrm{c} \alpha_{3}}{\mu D}\right) \frac{F_{x}}{F_{z}} \\
& +\frac{\mu x_{4} \mathrm{c} \alpha_{3}-\mu x_{3} \mathrm{c} \alpha_{4}+h_{3} \mathrm{c} \alpha_{3} \mathrm{c} \alpha_{4}}{\mu D\left(\mathrm{c} \alpha_{3}-\mathrm{c} \alpha_{4}\right)} \\
\frac{M_{x}}{D F_{z}}= & -\frac{\mu}{\mathrm{s} \alpha_{3}-\mathrm{s} \alpha_{4}}\left(\frac{y_{4}-y_{3}}{D}+\frac{h_{3} \mathrm{~s} \alpha_{3}}{\mu D}\right) \frac{F_{y}}{F_{z}} \\
& +\frac{\mu y_{3} \mathrm{~s} \alpha_{4}-\mu y_{4} \mathrm{~s} \alpha_{3}-h_{3} \mathrm{~s} \alpha_{3} \mathrm{~s} \alpha_{4}}{\mu D\left(\mathrm{~s} \alpha_{3}-\mathrm{s} \alpha_{4}\right)}
\end{aligned}
$$

R-7

$$
\begin{aligned}
\frac{M_{y}}{D F_{z}}= & -\frac{\mu}{\mathrm{c} \alpha_{1}-\mathrm{c} \alpha_{3}}\left(\frac{x_{1}-x_{3}}{D}+\frac{h_{3} \mathrm{c} \alpha_{3}-h_{1} \mathrm{c} \alpha_{1}}{\mu D}\right) \frac{F_{x}}{F_{z}} \\
& +\frac{\mu x_{3} \mathrm{c} \alpha_{1}-\mu x_{1} \mathrm{c} \alpha_{3}+\left(h_{1}-h_{3}\right) \mathrm{c} \alpha_{1} \mathrm{c} \alpha_{3}}{\mu D\left(\mathrm{c} \alpha_{1}-\mathrm{c} \alpha_{3}\right)}
\end{aligned}
$$

$$
\begin{aligned}
\frac{M_{x}}{D F_{z}}= & -\frac{\mu}{\mathrm{s} \alpha_{1}-\mathrm{s} \alpha_{3}}\left(\frac{y_{3}-y_{1}}{D}+\frac{h_{1} \mathrm{~s} \alpha_{1}-h_{3} \mathrm{~s} \alpha_{3}}{\mu D}\right) \frac{F_{y}}{F_{z}} \\
& +\frac{\mu y_{1} \mathrm{~s} \alpha_{3}-\mu y_{3} \mathrm{~s} \alpha_{1}+\left(h_{3}-h_{1}\right) \mathrm{s} \alpha_{1} \mathrm{~s} \alpha_{3}}{\mu D\left(\mathrm{~s} \alpha_{1}-\mathrm{s} \alpha_{3}\right)}
\end{aligned}
$$

R-8

$$
\begin{aligned}
\frac{M_{y}}{D F_{z}}= & -\frac{\mu}{\mathrm{c} \alpha_{1}-\mathrm{c} \alpha_{4}}\left(\frac{x_{1}-x_{4}}{D}-\frac{h_{1} \mathrm{c} \alpha_{1}}{\mu D}\right) \frac{F_{x}}{F_{z}} \\
& +\frac{\mu x_{4} \mathrm{c} \alpha_{1}-\mu x_{1} \mathrm{c} \alpha_{4}+h_{1} \mathrm{c} \alpha_{1} \mathrm{c} \alpha_{4}}{\mu D\left(\mathrm{c} \alpha_{1}-\mathrm{c} \alpha_{4}\right)}
\end{aligned}
$$

$$
\begin{aligned}
\frac{M_{x}}{D F_{z}}= & -\frac{\mu}{\mathrm{s} \alpha_{1}-\mathrm{s} \alpha_{4}}\left(\frac{y_{4}-y_{1}}{D}+\frac{h_{1} \mathrm{~s} \alpha_{1}}{\mu D}\right) \frac{F_{y}}{F_{z}} \\
& +\frac{\mu y_{1} \mathrm{~s} \alpha_{4}-\mu y_{4} \mathrm{~s} \alpha_{1}-h_{1} \mathrm{~s} \alpha_{1} \mathrm{~s} \alpha_{4}}{\mu D\left(\mathrm{~s} \alpha_{1}-\mathrm{s} \alpha_{4}\right)}
\end{aligned}
$$

$$
\begin{aligned}
\frac{M_{y}}{D F_{z}}= & -\frac{\mu}{\mathrm{c} \alpha_{2}-\mathrm{c} \alpha_{3}}\left(\frac{x_{2}-x_{3}}{D}-\frac{h_{3} \mathrm{c} \alpha_{3}}{\mu D}\right) \frac{F_{x}}{F_{z}} \\
& +\frac{\mu x_{3} \mathrm{c} \alpha_{2}-\mu x_{2} \mathrm{c} \alpha_{3}-h_{3} \mathrm{c} \alpha_{2} \mathrm{c} \alpha_{3}}{\mu D\left(\mathrm{c} \alpha_{2}-\mathrm{c} \alpha_{3}\right)}
\end{aligned}
$$

R-9

$$
\begin{aligned}
\frac{M_{x}}{D F_{z}}= & -\frac{\mu}{\mathrm{s} \alpha_{2}-\mathrm{s} \alpha_{3}}\left(\frac{y_{3}-y_{2}}{D}+\frac{h_{3} \mathrm{~s} \alpha_{3}}{\mu D}\right) \frac{F_{y}}{F_{z}} \\
& +\frac{\mu y_{2} \mathrm{~s} \alpha_{3}-\mu y_{3} \mathrm{~s} \alpha_{2}+h_{3} \mathrm{~s} \alpha_{2} \mathrm{~s} \alpha_{3}}{\mu D\left(\mathrm{~s} \alpha_{2}-\mathrm{s} \alpha_{3}\right)}
\end{aligned}
$$

R-10

$$
\frac{M_{y}}{D F_{z}}=-\frac{\mu}{\mathrm{c} \alpha_{2}-\mathrm{c} \alpha_{4}}\left(\frac{x_{2}-x_{4}}{D}\right) \frac{F_{x}}{F_{z}}+\frac{\mu x_{4} \mathrm{c} \alpha_{2}-\mu x_{2} \mathrm{c} \alpha_{4}}{\mu D\left(\mathrm{c} \alpha_{2}-\mathrm{c} \alpha_{4}\right)}
$$

$$
\frac{M_{x}}{D F_{z}}=-\frac{\mu}{\mathrm{s} \alpha_{2}-\mathrm{s} \alpha_{4}}\left(\frac{y_{4}-y_{2}}{D}\right) \frac{F_{y}}{F_{z}}+\frac{\mu y_{2} \mathrm{~s} \alpha_{4}-\mu y_{4} \mathrm{~s} \alpha_{2}}{\mu D\left(\mathrm{~s} \alpha_{2}-\mathrm{s} \alpha_{4}\right)}
$$




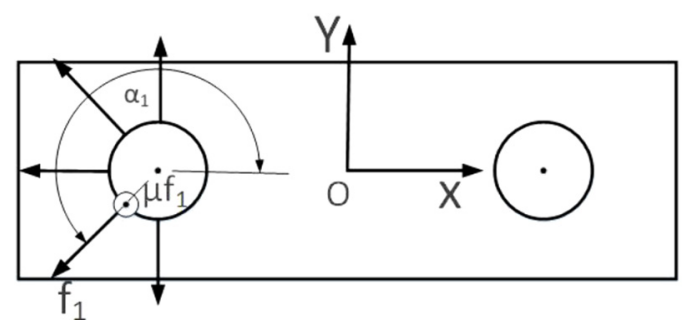

Fig. 8. Sectional view of the lower end face $X O Y$ of locating hole of the frame in the state L- $1 \alpha_{1} \in\left[90^{\circ}, 270^{\circ}\right]$.

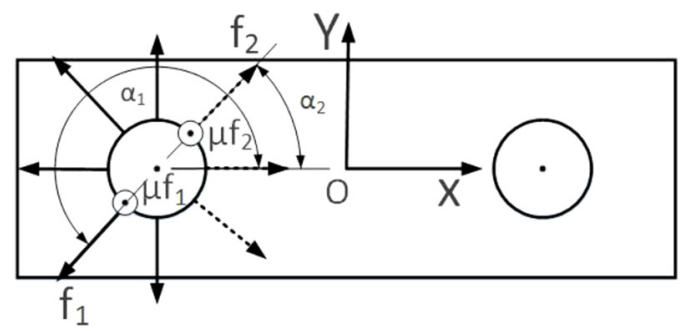

Fig. 9. Sectional view of the lower end face $X O Y$ of locating hole of the frame in the state L-5, $\alpha_{1} \in\left[90^{\circ}, 270^{\circ}\right], \alpha_{2} \in$ $\left[0^{\circ}, 90^{\circ}\right] \cup\left[270^{\circ}, 360^{\circ}\right]$.

(5) and (6) respectively show the corresponding linear relationship. For equation (5), when $\frac{F_{x}}{F_{z}}=-\frac{\cos \alpha_{1}}{\mu}$, the equation is reduced to equation (2), that is, $\frac{M_{y}}{F_{z}}=x_{1}$; when $\frac{F_{x}}{F_{z}}=-\frac{\cos \alpha_{2}}{\mu}, \frac{M_{y}}{F_{z}}=-\frac{h_{2} \cos \alpha_{2}}{\mu}+x_{2}$, as shown in Figure 10a. Similarly, for formula (6), when $\frac{F_{y}}{F_{z}}=-\frac{\sin \alpha_{1}}{\mu},(6)$ is reduced to formula (3), that is, $\frac{M_{x}}{F_{z}}=-y_{1}$; when $\frac{F_{y}}{F_{z}}=-\frac{\sin \alpha_{2}}{\mu}$, $\frac{M_{x}}{F_{z}}=\frac{h_{2} \sin \alpha_{2}}{\mu}-y_{2}$, as shown in Figure $10 \mathrm{~b}$ and c, where $\alpha_{1}$ $\in\left[90^{\circ}, 270^{\circ}\right], \alpha_{2} \in\left[0^{\circ}, 90^{\circ}\right] \cup\left[270^{\circ}, 360^{\circ}\right], r_{1}:\left(x_{1}, y_{1}, 0\right), r_{2}:\left(x_{2}\right.$, $\left.y_{2},-h_{2}\right)$. Thus, we can conclude that any two-point contact state is a combination of two one-point contact states. Based on these typical one-point contact analysis and two-point contact analysis and the relationship between assembly force and assembly moment under each contact condition in Table 2, we can draw three jamming diagrams as shown in Figure 10.

\section{Jamming analysis}

In the process of assembling the frame to the fixture, if there is relative position error between the locating hole and the locating pin, the proportion between the force and moment acting on the frame will be abnormal, which will lead to unsuccessful assembly. The jamming diagrams of the assembly of frame reflect the relationship between the force and the moment, which determines the frame can be correctly loaded into the fixture.

\subsection{Jamming diagram}

Due to the incorrect ratio between the assembly force and the assembly moment acting on the frame, the frame will be stuck outside the fixture. Through the relationship between $\frac{M_{y}}{F_{z}}$ and $\frac{F_{x}}{F_{z}}, \frac{M_{x}}{F_{z}}$ and $\frac{F_{y}}{F_{z}}$, three kinds of jamming diagrams can be made, as shown in Figure 10, in which Figure $10 \mathrm{~b}$ and $\mathrm{c}$ are derived since $\sin \alpha_{i}$ has a sign of positive and negative according to the range of value of $\alpha_{i}$. $\frac{M_{y}}{D F_{z}} \propto \frac{F_{x}}{F_{z}}$ represents the linear relationship between $\frac{M_{y}}{D F_{z}}$ and $\frac{F_{x}}{F_{z}}$, similarly, $\frac{M_{x}}{D F_{z}} \propto \frac{F_{y}}{F_{z}}$ represents the linear relationship between $\frac{M_{x}}{D F_{z}}$ and $\frac{F_{y}}{F_{z}}$.

Table 2 lists the conditions for the relationship between the forces and moments of the 20 contact states of the frame during assembly. Figure 10 draws three detailed jamming diagrams based on these contact states. When the force and moment conditions of all possible contact states are plotted in the force and moment space, the pattern will form a closed boundary, which divides the force and moment space into two spaces: statically unstable space and jamming space [2]. The unstable space is the area enclosed by the boundary. When the force and moment conditions fall into the closed area, the frame will be instability loaded into the fixture. The jamming space is the area outside the boundary, indicating that the frame will be stuck outside the fixture and the assembly will not be successful. Jamming diagrams can provide us with some information to ensure successful assembly. If the condition of assembly force and moment falls into the unstable space, the frame may fall into the locating hole of the fixture unsteadily and ensure the continuity of assembly. In actual production, since it is impossible to have all 20 contact states under specific geometric conditions, each jamming diagram representing a particular assembly problem is composed only of some of the straight lines shown in Figure 10. When the geometrical size and assembly depth of the frame are known, some possible states will be formed and corresponding jamming diagrams can be obtained, as shown in Figure 11.

\subsection{Eighteen kinds of possible jamming diagrams}

When the exact dimensions and assembly depth of the locating hole of the frame are known, the possible contact states can be obtained, and jamming diagrams can be constructed. These jamming diagrams provide us with information on how to apply assembly force and moment to the frame to achieve successful assembly. For the three jamming diagrams in Figure 10, each jamming diagram has 18 possible jamming diagrams. For example, if the geometric size and assembly depth of the frame are known, a possible jamming diagram can be formed by connecting four corresponding lines in Figure 10a to form a closed area, as shown in Figure 11. In Figure 11, the vertex of a quadrilateral represents one-point contact state, such as (L-1), (L-2), (R-1), (R-2) in Figure 11a, and the line of a quadrilateral represents two-point contact state, such as (L-5), (R-5) in Figure 11a.

In Figure 11, the vertical line describes the line contact state, and the combination of $F_{x}, F_{z}$, and $M_{y}$ falling on the quadrilateral indicates the movement in the equilibrium state; the combination outside the quadrilateral indicates that the frame is stuck outside the locating pin, whether 


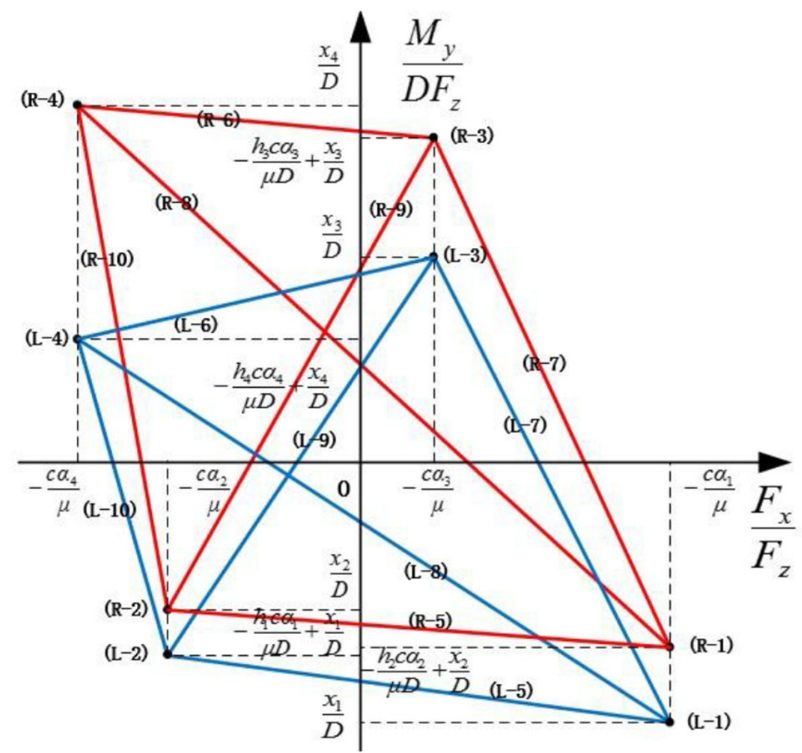

(a) $\frac{M_{y}}{D F_{z}} \propto \frac{F_{x}}{F_{z}}$

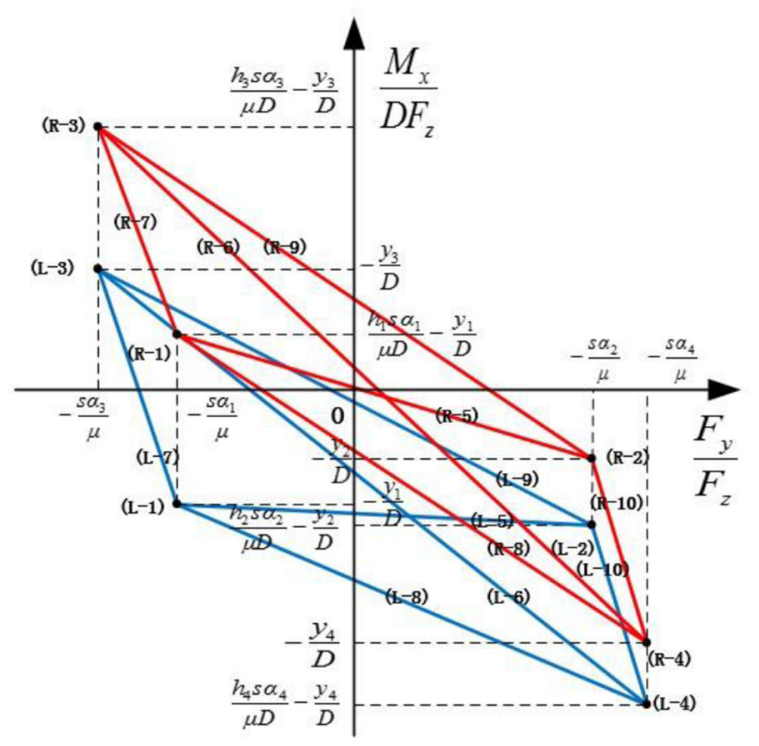

(b) $\frac{M_{x}}{D F_{z}} \propto \frac{F_{y}}{F_{z}}, \alpha_{1} \in\left[90^{\circ}, 180^{\circ}\right], \alpha_{3} \in\left[90^{\circ}, 180^{\circ}\right]$,

$\alpha_{2} \in\left[270^{\circ}, 360^{\circ}\right], \alpha_{4} \in\left[270^{\circ}, 360^{\circ}\right]$

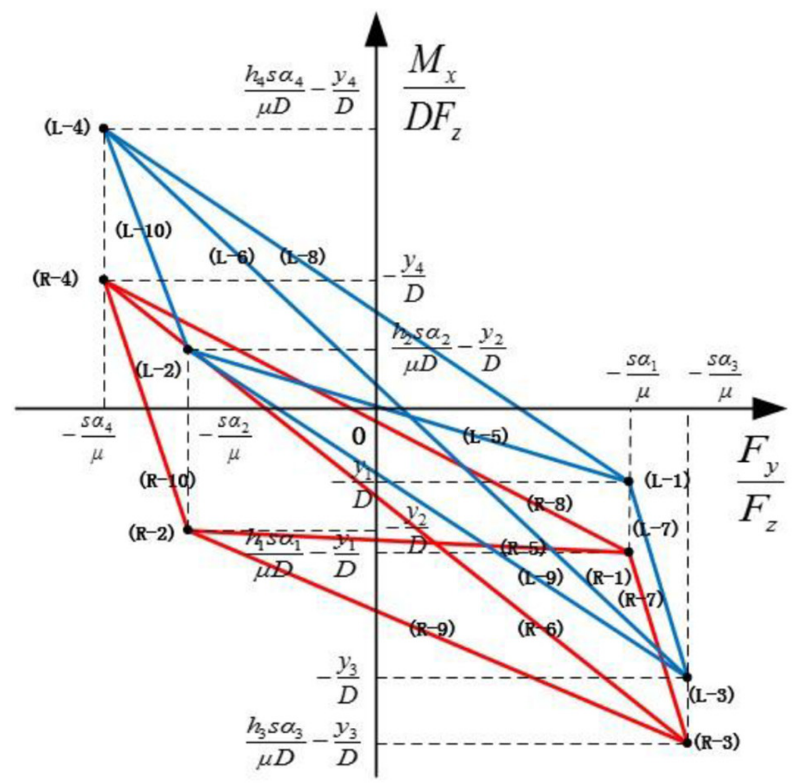

(c) $\frac{M_{x}}{D F_{z}} \propto \frac{F_{y}}{F_{z}}, \alpha_{1} \in\left[180^{\circ}, 270^{\circ}\right], \alpha_{3} \in\left[180^{\circ}, 270^{\circ}\right]$,

$\alpha_{2} \in\left[0^{\circ}, 90^{\circ}\right], \alpha_{4} \in\left[0^{\circ}, 90^{\circ}\right]$

Fig. 10. Jamming diagrams.

one-point contact or two-point contact; inside, the frame can be properly loaded into the locating pin and ensure successful assembly.

\section{Assembly strategy analysis}

There are three stages in the assembly process of automobile alternator frame: free stage, contact stage and mated stage, as shown in Figure 12, where $P_{1}$ and $P_{2}$ represent the lowest and highest points of the two locating holes on frame respectively. In actual assembly, due to the limitation of assembly conditions and geometric conditions of automobile alternator frame and fixture, the most possible jamming diagram can be selected to guide the assembly.

For the different assembly stages shown in Figure 12, different assembly control strategies are adopted. 


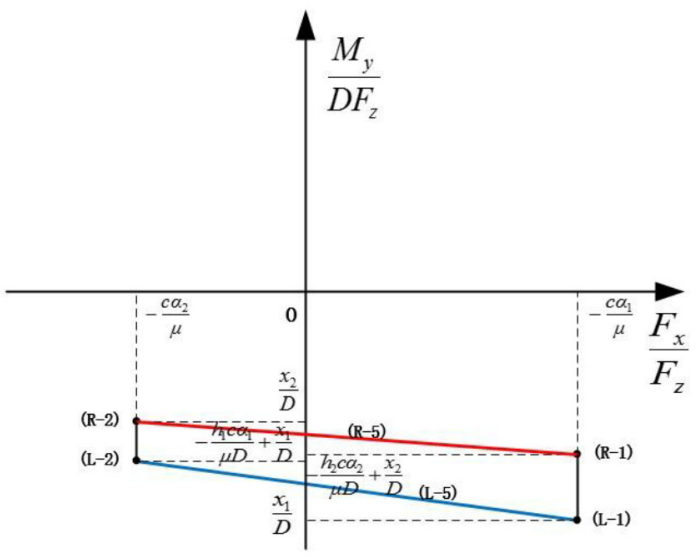

(a) L-5 and R-5

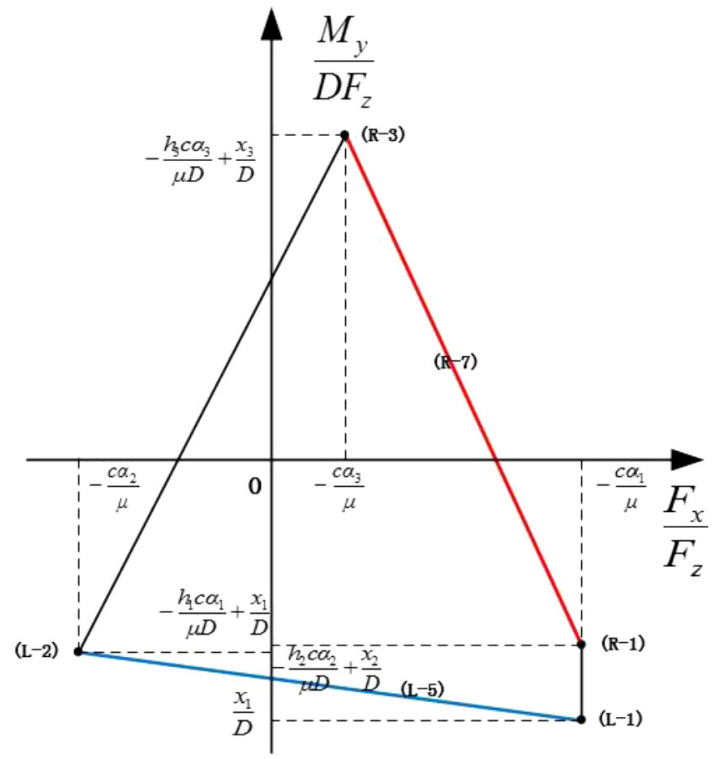

(c) L-5 and R-7

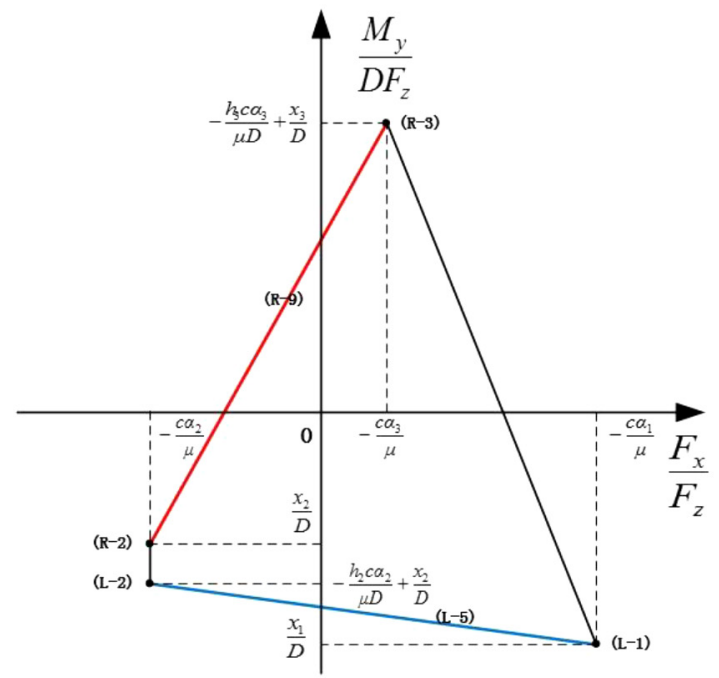

(e) L-5 and R-9

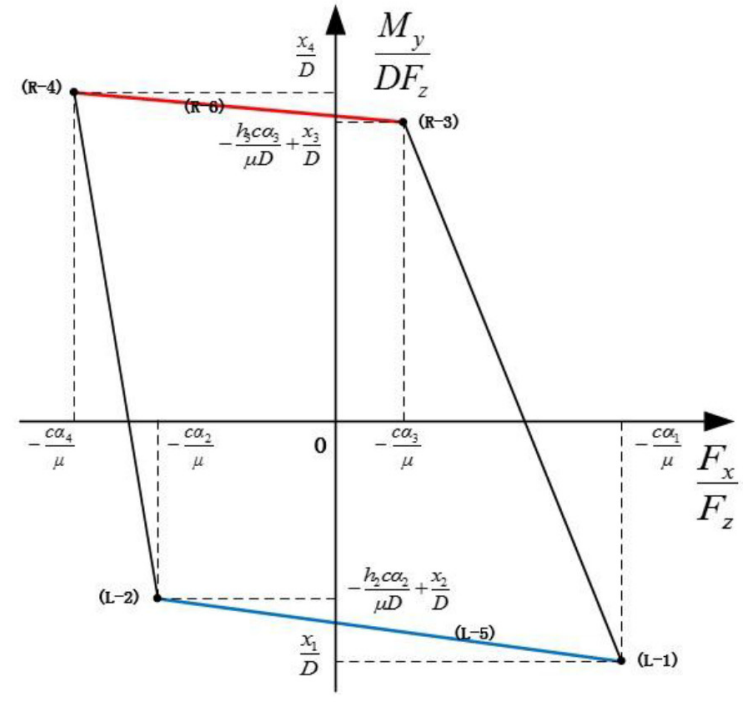

(b) L-5 and R-6

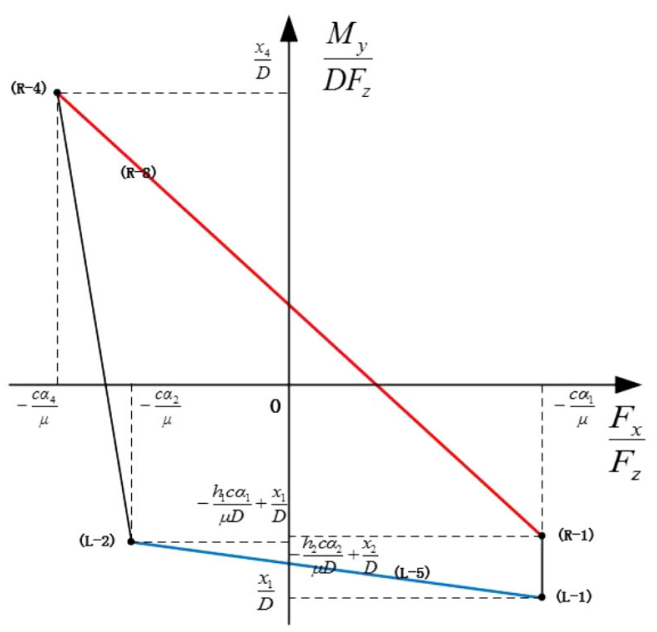

(d) L-5 and R-8

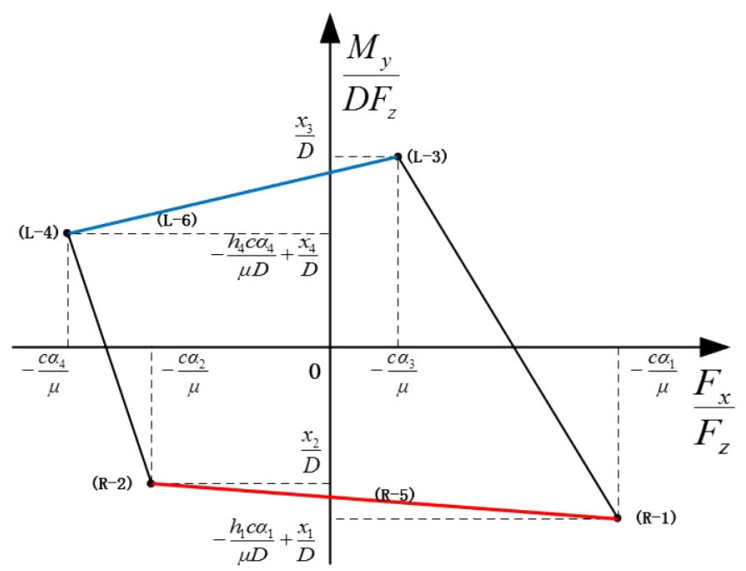

(f) L-6 and R-5

Fig. 11. 18 kinds of jamming diagrams $\left(\frac{M_{y}}{D F_{z}} \propto \frac{F_{x}}{F_{z}}\right)$. 


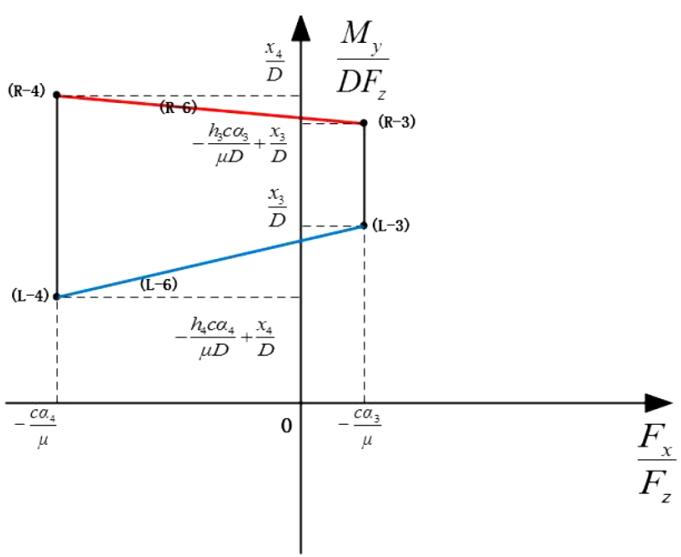

(g) L-6 and R-6

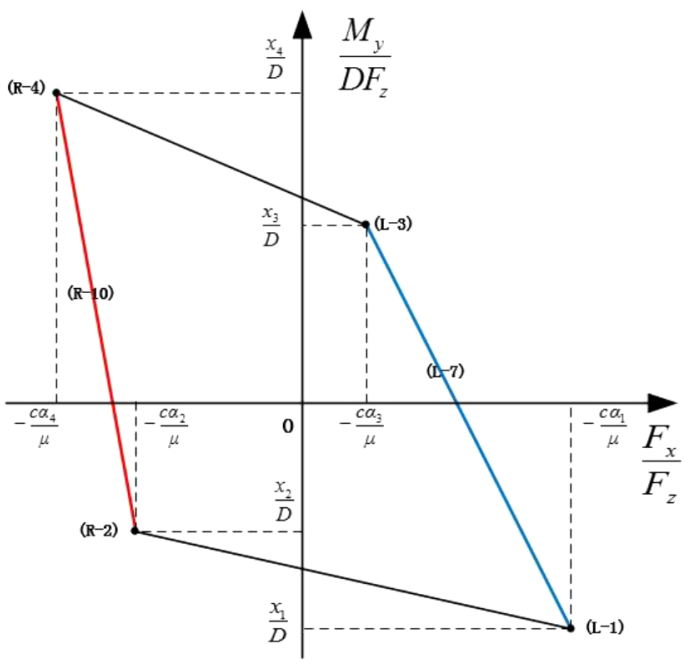

(i) L-7 and R-10

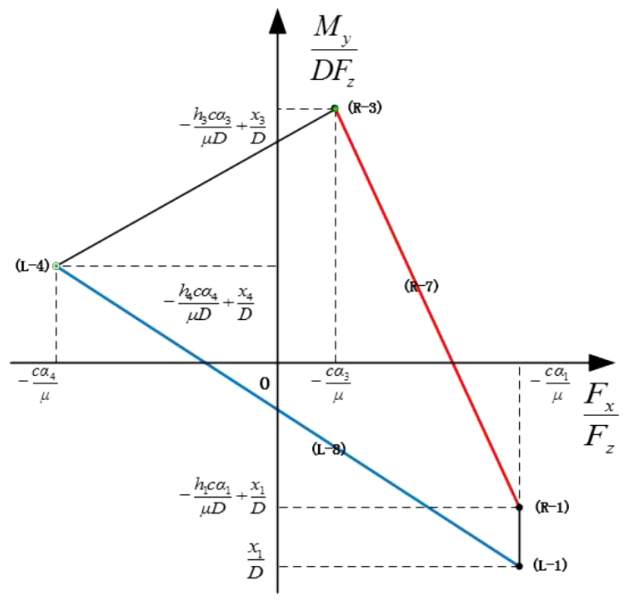

(k) L-8 and R-7

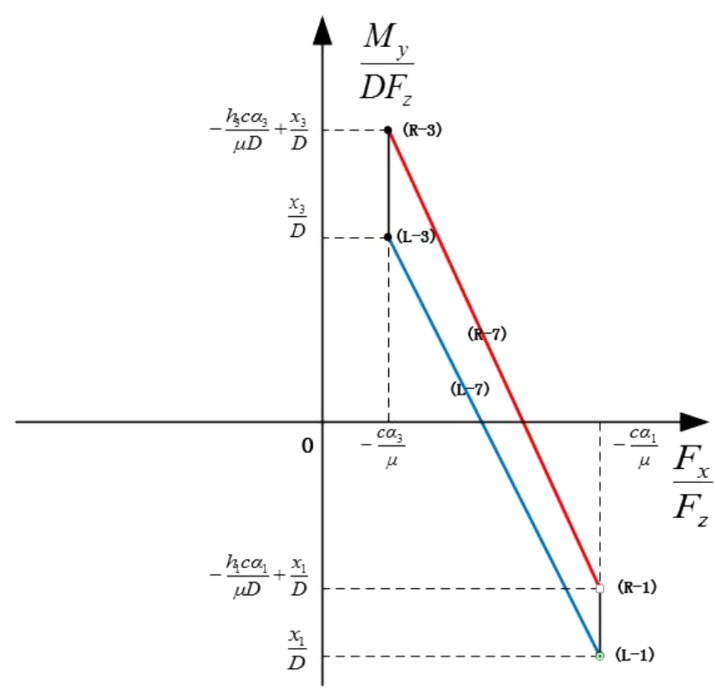

(h) L-7 and R-7

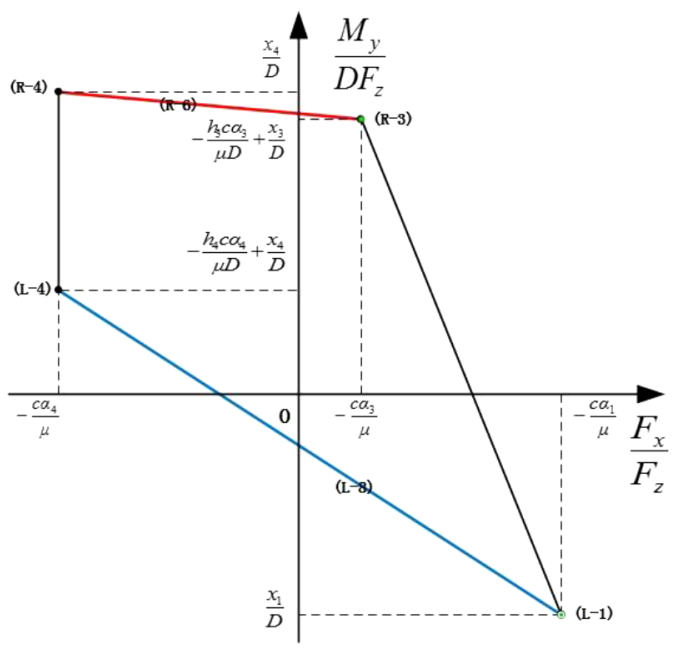

(j) L-8 and R-6

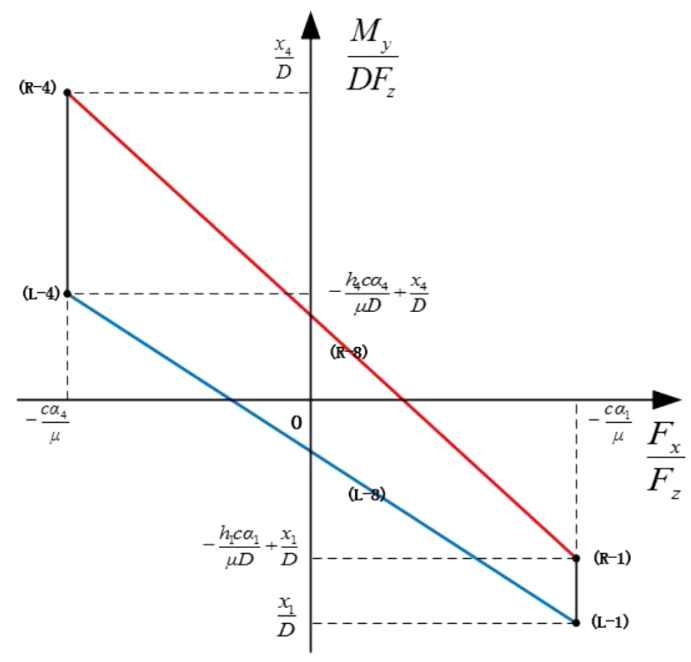

(1) L-8 and R-8

Fig. 11. continued. 


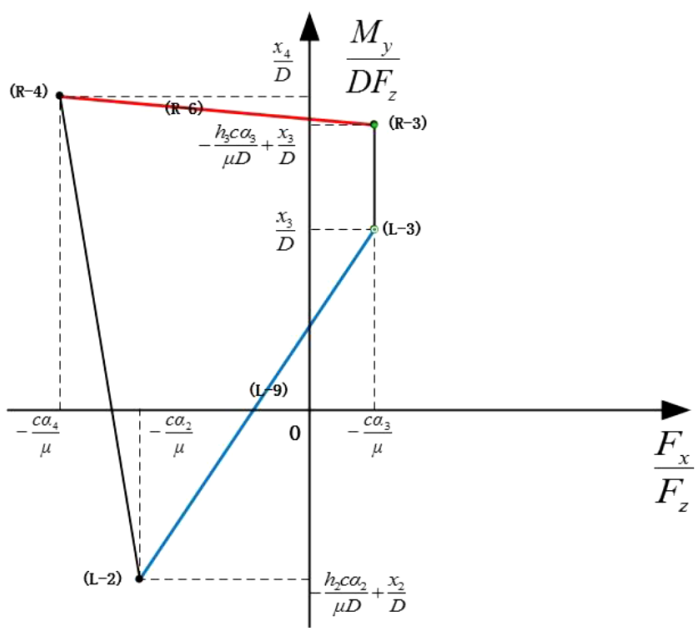

(m) L-9 and R-6

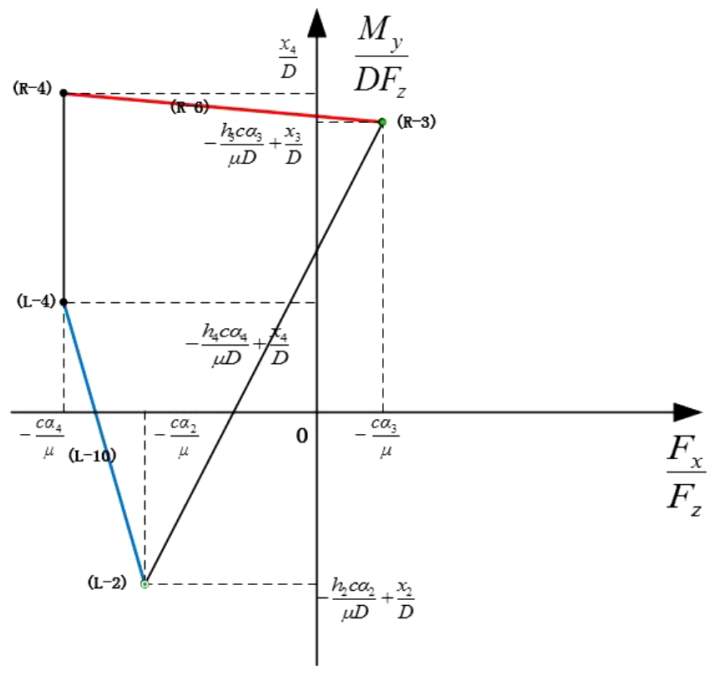

(o) L-10 and R-6

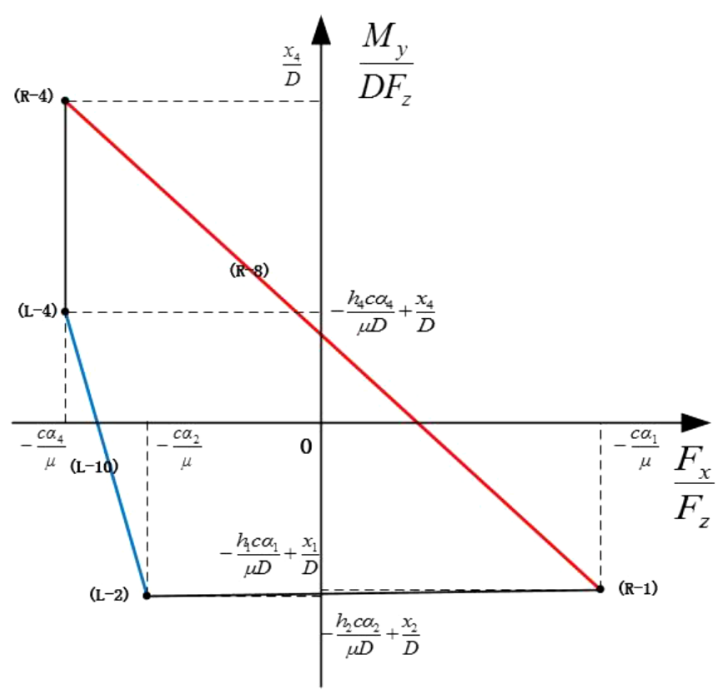

(q) L-10 and R-8

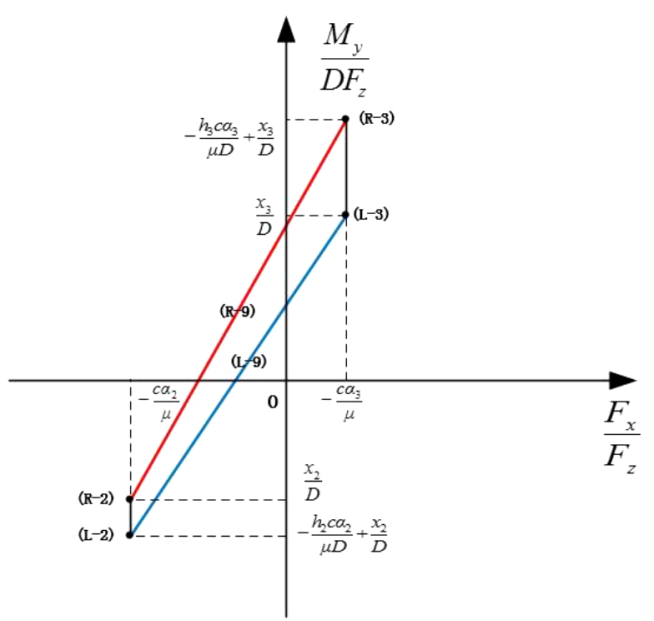

(n) L-9 and R-9

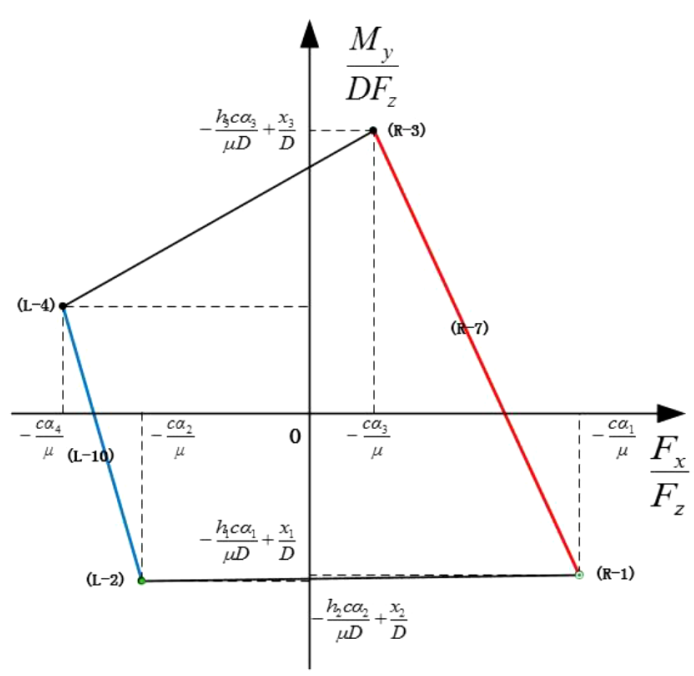

(p) L-10 and R-7

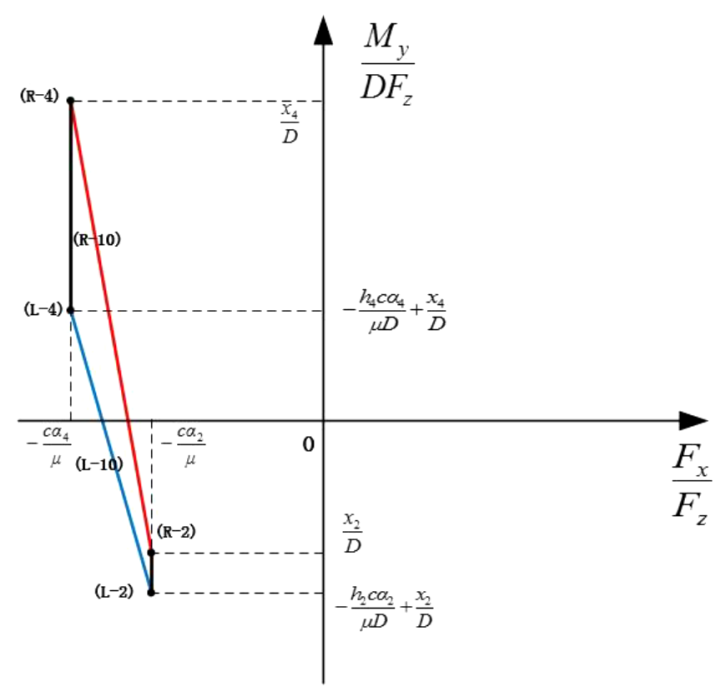

(r) L-10 and R-10

Fig. 11. continued. 


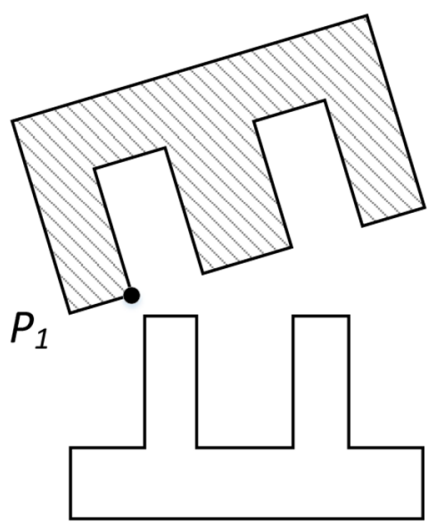

(a) free stage

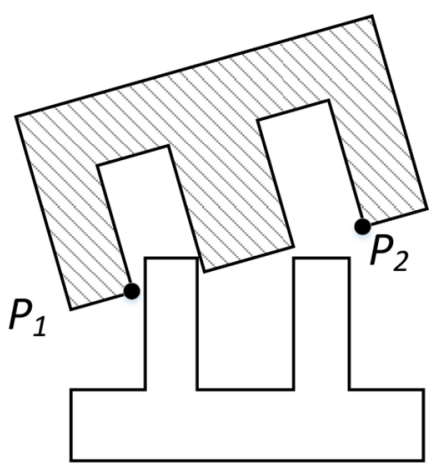

(b) contact stage

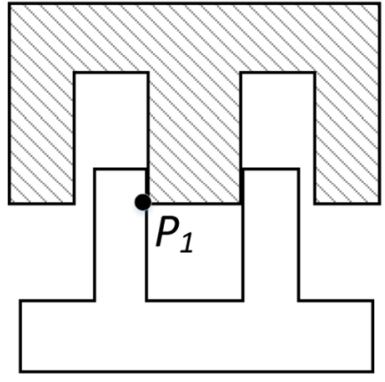

(c) mated stage

Fig. 12. Assembly stages of automobile alternator frame.

- Free stage: the control strategy of this assembly stage is position control. The contact force threshold should be set in the vertical direction to avoid the automobile alternator frame being scratched to the fixture. When the contact force exceeds the threshold, the automobile alternator frame stops moving.

- Contact stage: the control strategy of this stage is forceposition and velocity control. This control method is also called impedance control, which is very common in robot control. As shown in Figure 13a, the real-time positions of the bottom center of the two locating holes of automobile alternator frame relative to the fixture are $x(t), y(t)$ and $z(t)$, and their increments are $d x(t), d y(t)$ and $d z(t)$. The speed of automobile alternator frame is $v(t)$, and the preset reference force and the real-time force measured by the six-axis force sensor are $F_{0}$ and $F$ $(t)$, respectively. The real-time error of $F(t)$ is $e_{f}(t)$, and the differential of $e_{f}(t)$ is $d e_{f}(t) / d t . K_{v}, K_{d z}, K_{p z}, K_{p x y}$ and $K_{\mathrm{d} x y}$ are preset coefficients of the control system. Their values are determined according to the actual situation, and $J_{f}$ is jamming factor. In Figure $13 \mathrm{~b}$, point $J$ represents the jamming point, $l_{2}$ represents the distance from the jamming point to the zero point, and $l_{1}$ represents the distance from the corresponding point on the jamming diagram to the zero point. With this impedance control method, the position and speed of the automobile alternator frame can be controlled by force. The reference force and moment are set in advance, and compared with the force and moment collected by the six-dimensional force sensor. When there is a error between them, the error is used to guide the framer to move continuously until the frame positioning hole and fixture positioning pin are matched.

- Mated stage: the control strategy in this stage is still force-position and velocity control. The reference force in the vertical direction will increase, and the reference force in other directions will be set to zero. Finally, automobile alternator frame will move along the fixture positioning pin with the minimum contact force to complete the assembly process.
In the contact and mated stages, jamming factor should be determined according to selected jamming diagram. $J_{f} K_{p z}$ and $J_{f} K_{p x y}$ are used to control the movement of the alternator frame. If the alternator frame is in the jamming state, $J_{f}$ will be reduced, then $J_{f} K_{p z}$ will make the frame move more slowly in the $Z$ direction to avoid the jamming state in the next step, and $K_{p x y} / J_{f}$ will control the frame to move more quickly in the $X$ and $Y$ directions to get rid of the jamming state. Jamming factor $J_{f}$ is determined by the ratio between $l_{1}$ and $l_{2}$. If the point is outside the jamming diagram and $l_{2}$ is greater than $l_{1}$, then the jamming factor is $J_{\text {finin }}+\left(1-J_{\text {fimin }}\right)\left(l_{1} / l_{2}\right)$, otherwise the jamming factor is 1 . If $l_{2}$ tends to infinity, $J_{f}$ will be $J_{\text {finin }} . J_{\text {fimin }}$ is the minimum value of the jamming factor, which can be selected and determined according to the actual situation.

\section{Experiment analysis}

The experimental platform is shown in Figures 1 and 2. A YASKAWA robot and a 6-DOF force/moment sensor are used in the experiment. In this experiment, the pneumatic hand grasp at the end of the robot can grab the automobile alternator frame, while the fixture is fixed on the platform of the drilling and milling machining center. The parameters of the assembly of the frame are shown in Table 3, and the meanings of the parameters in Table 3 are shown in Figure 5.

\subsection{Experimental design}

As can be seen from Figure 13a, there are five control system parameters $K_{v}, K_{d z}, K_{p z}, K_{p x y}$ and $K_{\mathrm{d} x y}$ in this assembly control method. In the contact and mated stage, the control strategy is force-position and velocity control. $K_{p z}=0.001, K_{p x y}=0.0001, K_{d z}=0.0001, \quad K_{d x y}=0.00001$ and $K_{v}=1$. The selected minimum value of the jamming factor is $J_{\text {finin }}=0.5$. 


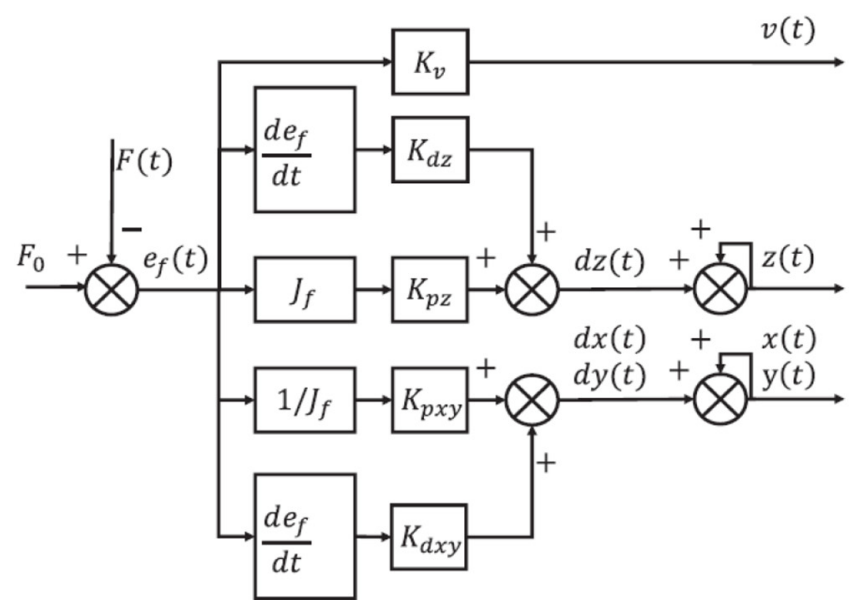

(a) force-position and velocity control

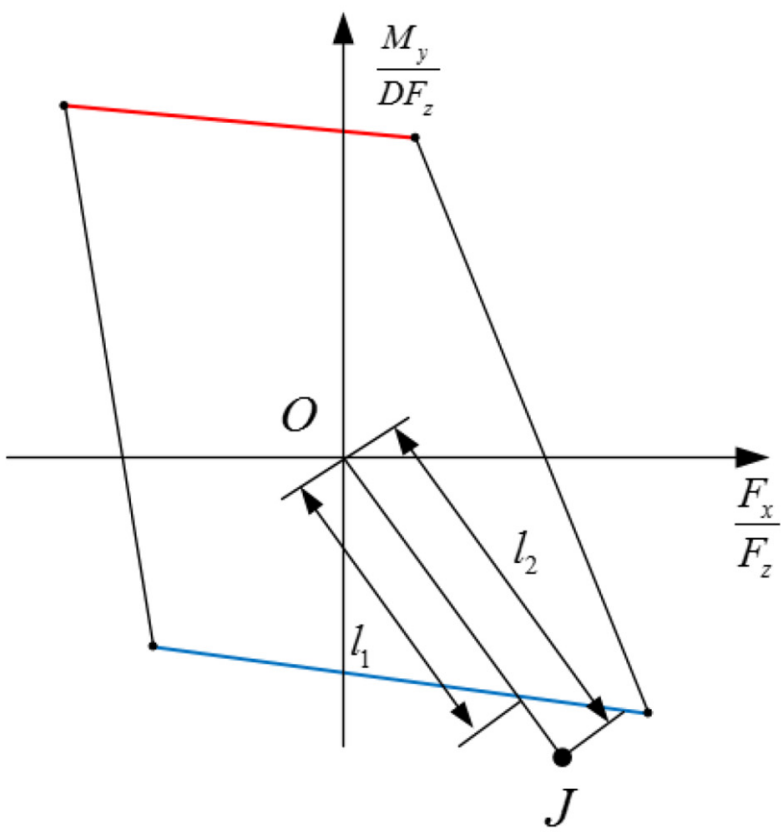

(b) jamming point and jamming diagram

Fig. 13. Control strategy and jamming factor.

Table 3. Assembly paramaters of the frame.

\begin{tabular}{llll}
\hline Frame & $r_{H_{1}}=3.5 \mathrm{~mm}$ & $r_{H_{2}}=4.5 \mathrm{~mm}$ & $D_{H}=94.5 \mathrm{~mm}$ \\
Fixture & $r_{p_{1}}=3 \mathrm{~mm}$ & $r_{p_{2}}=4 \mathrm{~mm}$ & $D\left(D_{p}\right)=94 \mathrm{~mm}$ \\
\hline
\end{tabular}

In the contact state, reference forces $\left[F_{x}, F_{y}, F_{z}\right]=[0,0$, $50] \mathrm{N}\left[M_{x}, M_{y}, M_{z}\right]=[0,0,0] \mathrm{N} \cdot \mathrm{m}$. In the mated state, reference forces $\left[F_{x}, F_{y}, F_{z}\right]=[0,0,160] \mathrm{N}\left[M_{x}, M_{y}, M_{z}\right]=[0$, $0,0] \mathrm{N} \cdot \mathrm{m}$. These reference forces will be compared with the forces acquired by the six-dimensional force sensor to adjust and control the motion of the robot.

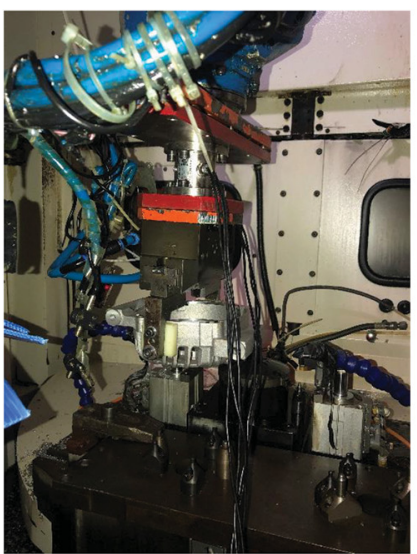

(a)

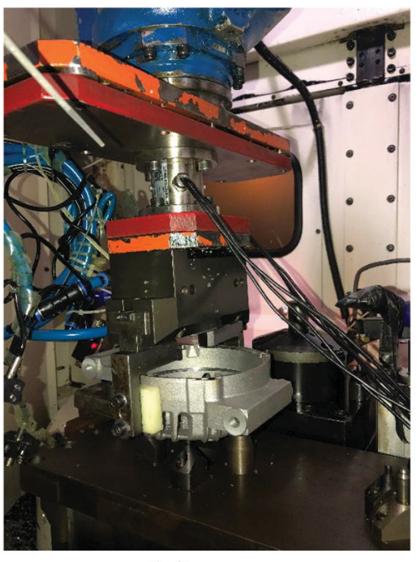

(c)

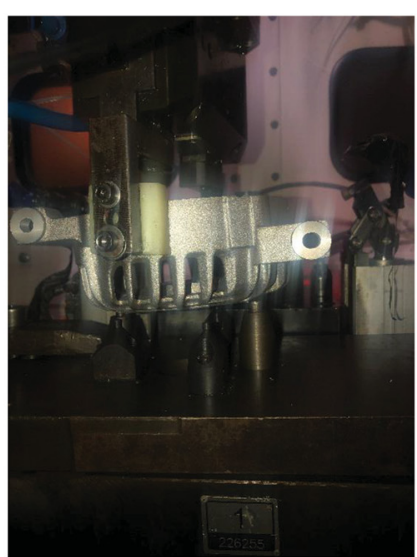

(b)

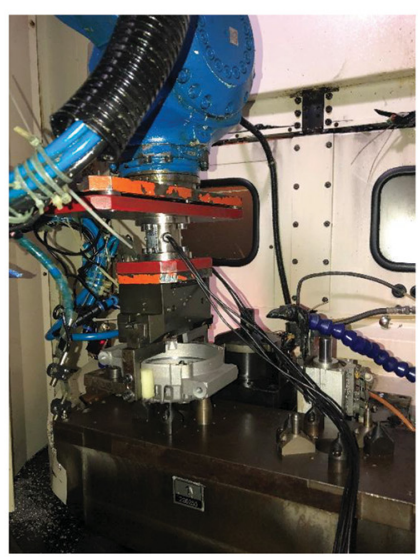

(d)
Fig. 14. Assembly process of automobile alternator frame.

\subsection{Experimental result}

The assembly experiment of automobile alternator frame was successfully completed. As shown in Figure 14, the automobile alternator frame moved from the free state (a) to the contact state (b) and the mated state (c), then the assembly depth of the positioning hole of the alternator frame increased continuously, and finally the whole assembly process was completed (d).

The position and force/moment data are shown in Figures 15 and 16 . The position of the automobile alternator frame changes slowly at the beginning, and the slope of coordinate curve is about $0.035 \mathrm{~mm} / \mathrm{step}$ (see Fig. 15). At this time, the resistances between automobile alternator frame and fixture are larger such as $F_{z}=$ $45.770 \mathrm{~N}$ and $M_{x}=-8.397 \mathrm{~N} \cdot \mathrm{m}$ because of the position error and jamming state (see Fig. 16).

After translating and rotating, the position error of automobile alternator frame decreases, the slope of coordinate curve increases to $0.139 \mathrm{~mm} / \mathrm{step}$ (see Fig. 15), and $F_{z}$ decreases to $9.007 \mathrm{~N}$ (see Fig. 16), which means that the contact resistances between the frame and the fixture decrease, and the frame begins to mate with the fixture.

Then, in the contact stage and the mated stage of the assembly process of the automobile alternator frame, the 


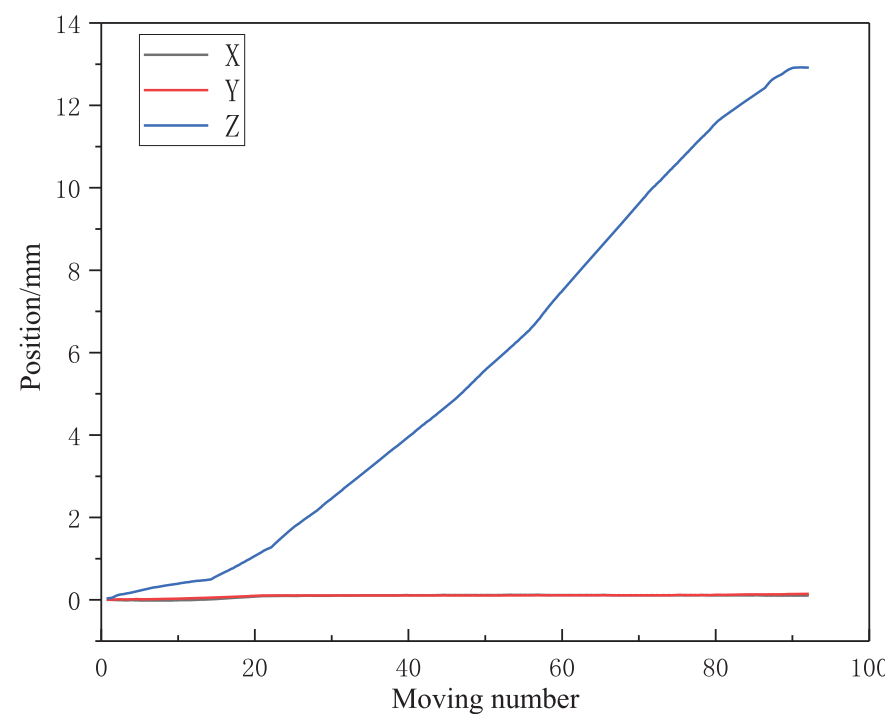

Fig. 15. Position of frame relative to fixture coordinate system.

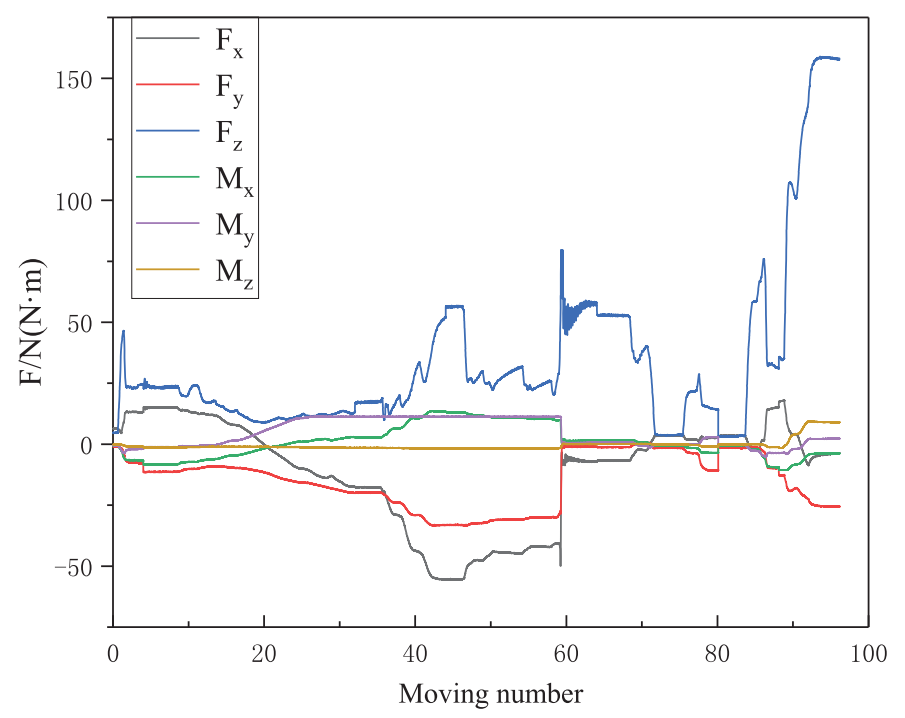

Fig. 16. Six-dimensional force data.

assembly forces and moments of some states are selected according to a certain moving sequence number interval. From the relationship between these forces and moments and the selected jamming diagram, the experimental jamming diagram in the assembly process can be drawn as shown in Figure 17. It can be seen that $70.59 \%$ of the points fall inside the quadrilateral, which means that the overall assembly process is relatively smooth, but there are also $29.41 \%$ of the points falling outside the quadrilateral. At this time, the alternator frame is in a jamming state. Then the jamming factor $J_{f}$ would adjust to control the movement of the alternator frame to successfully complete the assembly.

The jamming factor is used to assist the control of the movement of the alternator frame, which can avoid the stuck and wedge of the frame. For example, at the

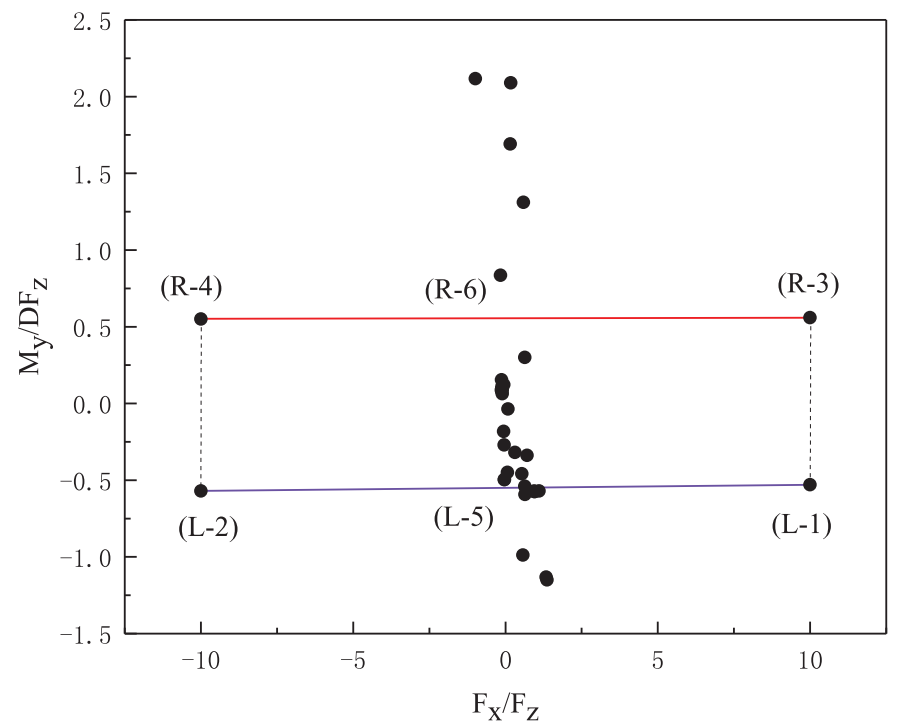

Fig. 17. Experimental jamming diagram.

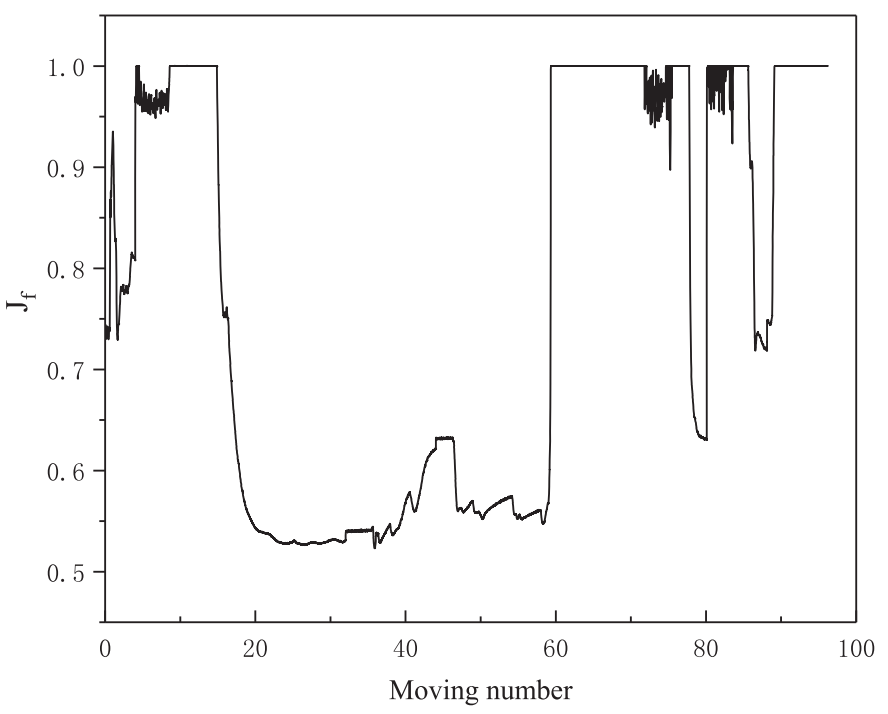

Fig. 18. The change of $J_{f}$.

beginning of assembly (moving number 2), the alternator frame is in contact jamming state, and the jamming factor is reduced to 0.767 . Then, the assembly force and moment are significantly reduced: $F_{z}$ is reduced from $45.77 \mathrm{~N}$ to $23.22 \mathrm{~N}$, and $M_{y}$ is reduced from -3.606 to -2.172 . The variation of jamming factor during assembly is shown in Figure 18. Overall, assembly force and moment decrease with the decrease of jamming factor, and the variation trend of assembly force and moment is similar to that of jamming factor, which also verifies the validity of the previous analysis.

\section{Conclusion}

- For the assembly contact problems in picking and placing the frame in multi-station processing of the automobile 
alternator frame, the specific three-dimensional assembly characteristics of the frame and fixture in milling, drilling and tapping positions are analyzed in detail, and the whole assembly is simplified and modeled. The simplified result is a dual peg-in-hole assembly, in which the alternator frame with two locating holes is simplified as a part with holes, and the fixture with two locating pins and three supporting pins is simplified as a part with shafts.

- After the simplification, the contact state analysis is carried out, and all 20 possible one-point contact states and two-point contact states (L-1) (R-10) are analyzed. Multi-point contact states with more than two points could not be sustained, so they are ignored in this analysis.

- The contact force analysis is carried out for all contact states, and it is concluded that in the typical one-point contact state L-1, $\frac{F_{x}}{F_{z}}=-\frac{\cos \alpha_{1}}{\mu}, \frac{M_{y}}{F_{z}}=x_{1}, \frac{F_{x}}{F_{z}}=-\frac{\cos \alpha_{2}}{\mu}$, and $\frac{M_{y}}{F_{z}}=-\frac{h_{2} \cos \alpha_{2}}{\mu}+x_{2}$, and the other contact states have the same form.

- One of the typical two-point contact states is analyzed, and the relationship between the force and the moment shown in formulas (5) and (6) is obtained. Similarly, the other two-point contact states have similar relations, as shown in Table 2. It is further concluded that any twopoint contact state is a linear combination of two onepoint contact states, which can be seen from three jamming diagrams (see Fig. 10), and 18 specific jamming diagrams are obtained.

- Experiments are carried out to verify the results of the previous analysis. The results confirm the validity of the front force analysis and jamming analysis, which provides theoretical and technical support for the assembly of automobile alternator frames based on force sensing control for the follow-up work.

Acknowledgements. The authors gratefully acknowledge the financial support of the ningbo industrial major project (No. 2017B10027).

\section{References}

[1] K. Sathirakul, R.H. Sturges, Jamming conditions for multiple pegin-hole assemblies, Robotica 16, 329-345 (1998)

[2] T. Arai, Hole search planning for peg-in-hole problem, Manufactur. Syst. 26, 119-124 (1997)

[3] R. Sturges, A three-dimensional assembly task quantification with application to machine dexterity, Int. J. Robot. Res. 7, 34-78 (1988)

[4] R. Sturges, S. Laowattana, Virtual wedging in three-dimensional peg insertion tasks, J. Mech. Des. 118, 99-105 (1996)

[5] F. Yanqiong, Z. Xifang, Contact and jamming analysis for three dimensional dual peg-in-hole mechanism, Mech. Mach. Theory 39, 477-499 (2004)

[6] Z. Kuangen, X. Jing, C. Heping et al., Jamming analysis and force control for flexible dual peg-in-hole assembly, IEEE Trans. Ind. Electr. 66, 1930-1939 (2019)

[7] Z. Kuangen, S. Minhui, X. Jing et al., Force control for a rigid dual peg-in-hole assembly, Assem. Autom. 37, 200-207 (2017)

[8] L. Renjiang, C. Yang, Study on simulation optimization problem of flexible manufacturing scheduling based on Petri net and Flexsim, Manufactur. Autom. 11, 68-72 (2015)

Cite this article as: B. Zhou, L. Liu, G. Chen, Contact analysis for dual peg-in-hole assembly of automobile alternator frame, Mechanics \& Industry 21, 209 (2020) 